\title{
SHRIMP U-Pb Geochronology of the Socorro Batholith and implications for the Neoproterozoic evolution in SE Brazil
}

\author{
Bárbara Bueno Toledo'* (D), Valdecir de Assis Janasi ${ }^{1}$ (D), Luiz Gustavo Ribeiro da Silva ${ }^{1}$
}

\begin{abstract}
The Socorro Batholith is one of the most expressive granite manifestations associated with the Neoproterozoic evolution in SE Brazil, occupying large areas $\left(-1,200 \mathrm{~km}^{2}\right)$ in the southern portion of the Socorro-Guaxupé Nappe. A U-Pb zircon SHRIMP dating program was developed to determine the ages of the main components of this batholith, identified in previous detailed mapping projects. High-K calc-alkaline (HKCA) porphyritic biotite-hornblende granites with relatively low (60-67 wt\%) SiO are the most voluminous component of this and other large "syn-tectonic" batholiths in the SGN (Água Limpa and Pinhal-Ipuiuna) and neighboring domains located south of it in the Apiai and São Roque Domains of the Ribeira Fold Belt. Two samples collected in widely separated localities at the northern and southern part of the Socorro batholith yield similar ages of magmatic crystallization, respectively $610.1 \pm 7.0$ and $608.3 \pm 6.6 \mathrm{Ma}$. A more fractioned (> $72 \mathrm{wt} \% \mathrm{SiO}$ ) granite reported in the literature as related to a younger event ("Socorro II magmatism", as opposed to the previous "Socorro I" HKCA granites) yield a precise age that is clearly older (624.4 $\pm 3.6 \mathrm{Ma}$ ), and contemporary to anatectic granites and migmatites that were produced during a prolonged period of high-grade metamorphism (635-605 Ma) that affected the SGN. Our data thus indicates that at least part of the HKCA magmatism that constitutes the Socorro batholith post-dates the high-P metamorphism associated to continental collision, and may have been a source of heat and volatiles to the high-T metamorphism responsible for partial melting of the upper portions of the crustal section represented by the SGN. Two charnockitic rocks that show transitional contacts with granites of the Socorro batholith were also dated. The Socorro Charnockite is aged $641.6 \pm 4.1 \mathrm{Ma}$, which overlaps those of regional orthogneisses (in part also of charnockitic character) considered as associated with a pre-collisional tectonics (subduction-related?). However, it is reported to transition to granites that are very similar to the HKCA granites of the Socorro batholith, which are yet undated. The Atibaia Charnockite has distinct geochemical affinity (lower $m g \#$ and Sr content; higher $\mathrm{Zr}$ ), a younger age $(633.3 \pm 6.2 \mathrm{Ma}$ ), and may signal a different tectonic setting at the end of the period of plate consumption as yet poorly characterized.
\end{abstract}

KEYWORDS: zircon; U-Pb geochronology; SHRIMP; Socorro-Guaxupé Nappe; High-K calc-alkaline granite.

\section{INTRODUCTION}

One of the foremost features of the Neoproterozoic evolution of SE Brazil is the generation of voluminous granite magmatism of widely varied composition, whose ages spread for a large time interval ( $-800-500 \mathrm{Ma})$. Metaluminous, porphyritic (hornblende)-biotite granitoids (dominantly monzogranites, quartz monzonites and granodiorites) with I-type, high-K calc-alkaline (HKCA) character, are by far the most abundant granite type, and are the main components of some extensive batholithic masses with areas over $2,000 \mathrm{~km}^{2}$ (Fig. 1). Although the tectonic significance of these HKCA granites is key to understanding the Neoproterozoic evolution of SE Brazil, their origin is still a matter of large uncertainties.
In fact, HKCA granites are known to be generated in various geodynamic environments (e.g., Barbarin 1999), occurring in both subduction-related and post-collisional settings. The occurrences in SE Brazil are usually associated in the literature to a continental-margin, subduction-related environment, in view of their huge volumes, deformed character and calc-alkaline character (e.g., Heilbron et al. 2004, 2017, Janasi \& Ulbrich 1991, Vinagre et al. 2014). However, some authors argue that the plutonic products of convergent tectonics may be restricted to older (> $640 \mathrm{Ma}$ ) granitoids that were largely converted to orthogneiss and migmatite (Hackspacher et al. 2003), and thus the bulk of the HKCA granites could be post-collisional (Meira et al. 2015). Precise dating and careful association with geological features related to plate-margin

${ }^{1}$ Instituto de Geociências, Universidade de São Paulo - São Paulo (SP), Brazil. E-mails: barbara.btoledo@usp.br, vajanasi@usp.br,guribeirova@gmail.com

*Corresponding author.

Manuscript ID: 20180040. Received on: 04/06/2018. Approved on: 08/16/2018. 
tectonics is thus key to correctly understanding the tectonic meaning of this magmatism.

Extensive geochronological surveys based on the U-Pb system in zircon and monazite by conventional TIMS have been carried out in some of the most expressive batholiths of SE Brazil, namely Três Córregos, Cunhaporanga (Gimenez Filho et al. 2000, Prazeres Filho et al. 2003) and Agudos Grandes (Janasi et al. 2001, Leite et al. 2007); more recently, in situ U-Pb dating of zircon by SHRIMP and LA-ICPMS was applied to the Serra da Água Limpa Batholith (Vinagre et al. 2014) and to the HKCA granites of the São Roque Domain (Janasi et al. 2016).

The Socorro Batholith is one of the most expressive occurrences of HKCA in SE Brazil, spreading out for over $1,200 \mathrm{~km}^{2}$ in the southern portion of the Socorro-Guaxupé Nappe, and was the subject of several mapping and geochemical studies in the 1980's that revealed a wide variety of granites which were grouped in different associations, including, apart from the predominant HKCA porphyritic hornblende-biotite granites, several types of fractioned, pink granites, and also locally charnockites (Artur et al. 1993, Campos Neto et al. 1984a, Wernick et al. 1984a, 1984b). However, the geochronology of the batholith is restricted to determinations by the Rb-Sr system, which clearly results in unreliable ages (e.g., Tassinari 1988), and to two U-Pb zircon dates by TIMS reported by Topfner (1996) (629 \pm $3 \mathrm{Ma})$ and Ebert et al. (1996) (610 $\pm 10 \mathrm{Ma})$, thus far considered as the best estimates of the ages of the predominant HKCA granite magmatism in the Socorro Batholith.

As part of an ongoing re-study of the Socorro Batholith, we selected five representative samples of the three main granite associations recognized in previous works for $\mathrm{U}-\mathrm{Pb}$ SHRIMP dating (Socorro I or Bragança Paulista association, corresponding to the predominant HKCA porphyritic hornblende-biotite monzogranite; Socorro II or Salmão, a pink, coarse-grained biotite syenogranite; and the Charnockite association; nomenclature by Artur et al. 1993 and Campos Neto et al. 1984a). Our results reveal a wide (> 30 M.y.) interval for the batholith construction, and suggest that the granite associations may have been generated in a succession that differs from what was previously admitted.

\section{GEOLOGICAL SETTING}

\section{Tectonic setting}

The Socorro-Guaxupé Nappe (SGN) is a giant allocthtonous terrane constituted by migmatites and (in its basal portions) granulites intruded by large volumes of granites in the Neoproterozoic, and is interpreted as the root of a magmatic arc that was thrust over the southern margin of the São Francisco Craton in the Ediacaran (Campos Neto \& Caby 2000). Current tectonic models admit that the SGN exposes a continuous section of middle to lower crust that records events related to convergent tectonics which evolved from subduction to continental collision and finally was involved in post-collisional transtensional tectonics and then intruded by post-orogenic granites of A-type character (the Itu Granitic Province; Janasi et al. 2009). The post-collisional transtensional tectonics may be a reflection of the development of a younger orogenic belt located to the SE (the Ribeira Fold Belt, part of the Mantiqueira Province, an extensive NeoproterozoicCambrian orogenic system running parallel to the Atlantic coastline in Brazil (Heilbron et al. 2004, 2017). Geologic terranes located immediately south of the SGN and related to the Ribeira Belt (Apiaí and São Roque domains) are also intruded by large volumes of "syn-tectonic" HKCA granites, and by post-orogenic granites of the Itu Granitic Province, which would thus straddle the limits between the SGN and Ribeira Belt. Some authors admit that the Apiaí and São Roque domains correspond, together with the SGN, to the reworked margin of a "cratonic" terrane (the Paranapanema lithospheric block of (Mantovani \& Brito Neves 2005). Domains located further E-SE in the Ribeira Belt (e.g., the Oriental terrane, Fig. 1) also bear significant volumes of Neoproterozoic granites, but the most voluminous "syn-tectonic" batholiths are typically younger (e.g., Heilbron et al. 2013), as is the post-orogenic granite magmatism (520-500 Ma; Valeriano et al. 2016).

The SGN is admitted by several authors to be related to the evolution of the southern portion of the Brasilia Belt (Campos Neto \& Caby 2000, Rocha et al. 2018). It would represent the active margin of Paranapanema Plate that was thrust over the passive margin of São Francisco paleocontinent (Campos Neto \& Caby 2000) in a collisional setting. Orthogneisses dated by Hackspacher et al. (2003) at 660-640 Ma would represent arc magmatism that resulted from subduction of Neoproterozoic oceanic crust during early precollisional convergence and closure of a branch of either the Adamastor or Goianides paleo-ocean.

\section{Neoproterozoic granitic magmatism in Socorro-Guaxupé Nappe}

Extensive elongated granitic batholiths of high-K calc-alkaline character are typical of both the Ribeira belt and the Socorro-Guaxupé Nappe. The tectonic context in which these rocks were formed is still ambiguous, being accepted as products of active continental margin magmatic arcs (Campos Neto et al. 1984a, Heilbron et al. 2004, 2013, 2017, Janasi \& Ulbrich 1991, Trouw et al. 2013), or considered belonging to a post-collision environment (Meira et al. 2015). 
Large volumes of crustal granites throughout the crustal section are exposed in the Socorro-Guaxupé Nappe. Temperatures close to $1,000^{\circ} \mathrm{C}$ were reached around $625 \mathrm{Ma}$ in the deeper portions of Socorro-Guaxupé Nappe (at $14 \mathrm{kbar}$ ), resulting in melting of depleted granulites and generation of charnockitic magmas (Janasi 2002). Similar age has been obtained for crustal granites generated at the temperature of biotite breakdown $\left(-850^{\circ} \mathrm{C}\right)$ by remelting of orthogneisses in the middle crust (biotite granite type Pinhal). At lower levels (in the southern portion of the Socorro Domain), garnet-biotite granites (Nazaré Paulista types), were generated by anatexis of paragneisses (or mixtures between ortho- and paragneisses), probably over a long period of time (ca. 25 M.y., between 635 and $610 \mathrm{Ma}$ ), as estimated by monazites dating in associated migmatites (Martins et al. 2009). Some large batholiths dominated by granitoids of high-K calc-alkaline occur in the SGN, PinhalIpuiúna Batnolith (Haddad 1995) in the northern portion, and Socorro (Artur et al. 1993) and Serra da Água Limpa batholiths (Vinagre et al. 2014) in the southern portion.

\section{Granites of Socorro Batholith}

The Socorro Batholith corresponds to an extensive area elongated in the $\mathrm{N} 30 \mathrm{E}$ direction in the southern portion of SGN dominated by granitic rocks (ca. $60 \times 25 \mathrm{~km}$; total ca. 1,200 km²) (Fig. 2).
The predominant lithology in the batholith is a porphyritic hornblende-biotite granite with up to $4 \mathrm{~cm}$ alkali feldspar megacrysts set in a medium to coarse-grained matrix, commonly foliated, with high color index (10-20), and monzogranitic to quartz monzonitic modal composition. These granites correspond to the Socorro I magmatism of Artur et al. (1993) and the Bragança Paulista suite of Campos Neto et al. (1984a) and are reported to be locally intruded by more felsic, pink to grey biotite granites, usually inequigranular, coarse to medium-grained, attributed to "Salmão" suite (Campos Neto et al. 1984a) or Socorro II magmatism (Artur et al. 1993). Small bodies of orthopyroxene-bearing, greenish granitoids (charnockites) occur in the northern portion of the batholith, where they are reported to transition into typical Socorro I granites (Artur 2003, Wernick et al. 1984a). Charnockites also form an isolated body in the southern part of the batholith, next to the city of Atibaia (Oliveira et al. 1994), where they seem to grade to pink, locally garnet-bearing granites.

Two U-Pb zircon ages are reported in the literature, and indicate ages between $629 \pm 3$ (Topfner 1996) and $610 \pm 10 \mathrm{Ma}$ (Ebert et al. 1996) for the predominant Socorro I granites; no reliable age determinations are available for the other granite types from the batholith. The country rocks of the batholith are orthogneisses and paragneisses usually affected by migmatization. U-Pb zircon TIMS ages reported for the orthogneisses

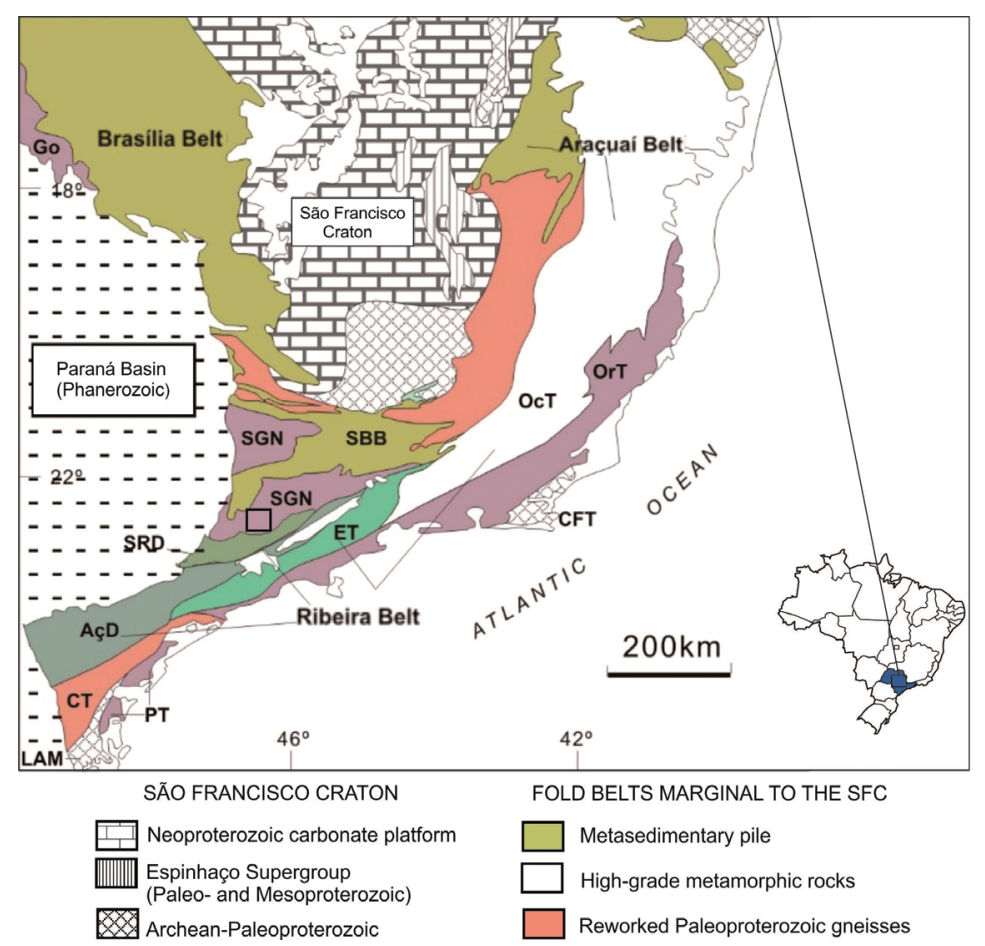

AçD: Açungui Doman; CFT: Cabo Frio Terrane; CT: Curitiba Terrane; ET: Embu Terrane; Go: Goiás Magmatic Arc; LAM: Luiz Alves Microplate; OcT: Occidental Terrane; OrT: Oriental Terrane; SBB: South Brasília Belt; SGN: Socorro-Guaxupe Nappe; SRD: São Roque Domain

Figure 1. Tectonic framework of SE Brazil with the main tectonic domains (Janasi et al. 2016). 
are in 660-640 Ma range (Hackspacher et al. 2003), and are seen as an upper limit to the ages of the Socorro granites. A recent LA-ICPMS dating program in the Serra da Água Limpa batholith, which seems to correspond to an eastern continuation of the Socorro Batholith, dated similar HKCA granites in the 645-630 Ma range (Vinagre et al. 2014).

The Socorro Batholith is intruded by post-orogenic granitic plutons, which are part of the Itu Granitic Province, constituted of granites of A-type or high-K calc-alkaline character and associated basic bodies (Janasi et al. 2009). These rocks may be envisaged as an "inboard reflection" of orogenic processes occurring at the Mantiqueira Orogenic System (Janasi et al. 2009). Besides the most voluminous bodies (Itu, Atibaia and Morungaba) the Guaripocaba stock is part of this province, and is intrusive in the area of the Socorro Batholith. The most robust ages for the Itu Province are ca. 580-570 Ma (Janasi et al. 2009), indicating that this magmatism occurred after a temporal hiatus, during which the SGN was raised.

\section{METHOD}

\section{Whole-rock major and trace-element analyses}

$\mathrm{X}$-ray Fluorescence was used to determine the major, minor and trace elements in whole rock samples. Large samples

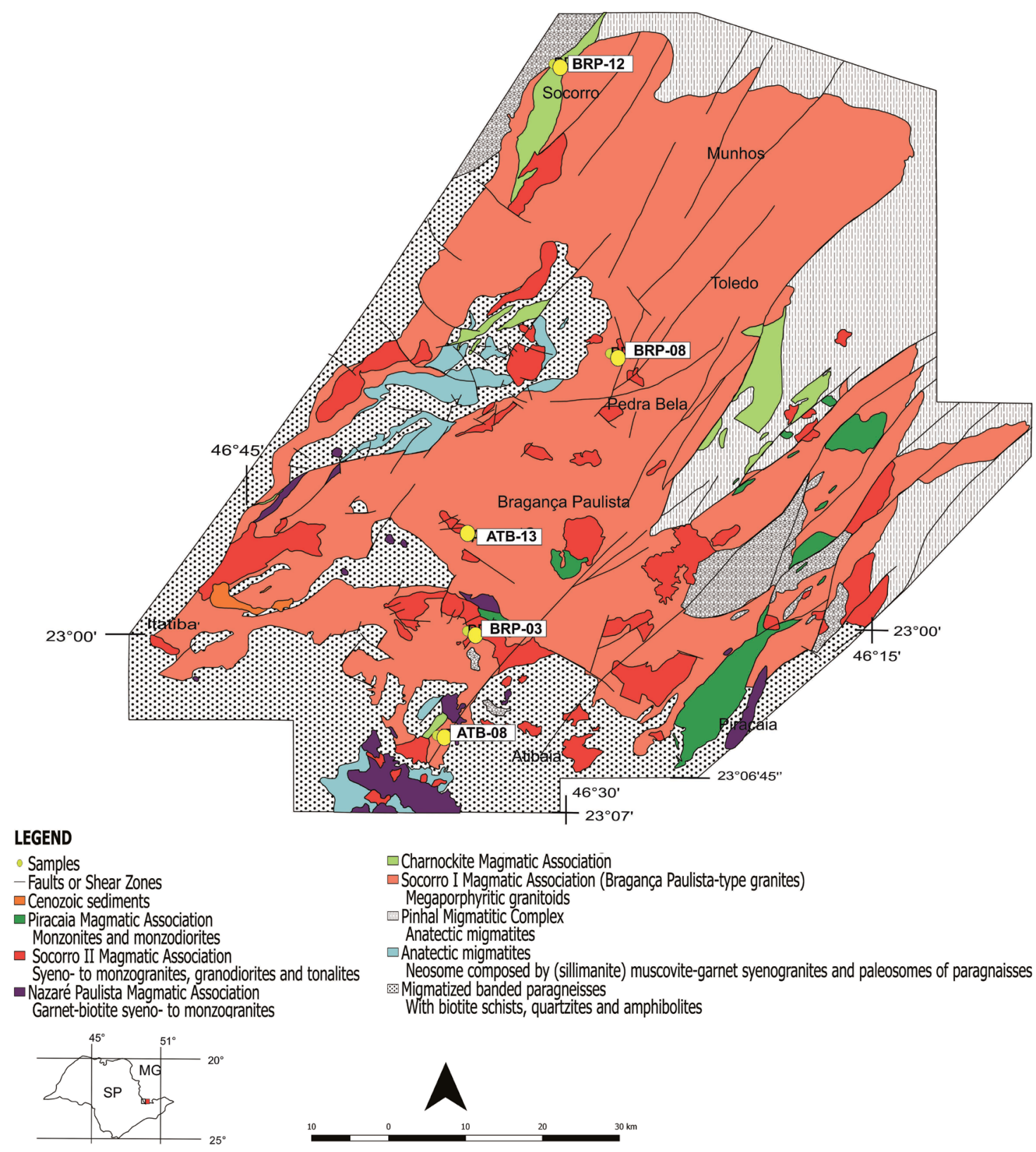

Figure 2. Geological map of the Socorro Batholith (modified from Artur 2003) with location of dated samples. 
typically weighing 6-8 $\mathrm{kg}$ were broken into pieces of about $3-5 \mathrm{~cm}$ using hammer or hydraulic press, and then were crushed to fragments with an average size of $1.5 \mathrm{~cm}$ with a jaw breaker of Mn-steel. The material was then split in a cross-type Jones splitter, yielding 100-150 g sub-samples representative of the starting material. The sub-samples were then milled for 15 minutes on a Pulverisette-type planetary agate mill. This overall time is usually enough for the material to reach a particle size smaller than 74 microns.

Analyses were performed in a PANalytical AXIOS MAX Advanced spectrometer at the NAP-Geonalitica, Instituto de Geociências, Universidade de São Paulo, Brazil, following the protocol described in Mori et al. (1999). Major and minor elements were analyzed from molten tablets obtained from $1 \mathrm{~g}$ sample and $9 \mathrm{~g}$ of a mixture of spectroscopic grade lithium tetra and metaborate at $1,100-1,200^{\circ} \mathrm{C}$ in a platinum crucible, using a Claisse fusion machine. Trace elements were analyzed from pressed pellets obtained from homogenization of the pulverized sample (previously micronized in a McCrone Micronizer) and pressing to 30 ton with wax. Analytical quality was controlled using a reference material granite JG-1a from Geological Survey of Japan as an unknown sample, and duplicated samples. The values obtained should be within $2 \sigma$ of the certified values, and the duplicated samples within $1 \%$ of variation.

Rare earth and other trace elements were determined by ICPMS in a Thermo-Analitica ICAP quadrupode analyzer at the NAP-Geonalitica, Instituto de Geociências, Universidade de São Paulo, Brazil, following the protocol described in Navarro et al. (2008).

Approximately $40 \mathrm{mg}$ of rock powder with grain size < 200 mesh were placed in a Teflon capsule Parr bomb and added with $5 \mathrm{~mL}$ of $\mathrm{HNO}_{3}$ and $15 \mathrm{~mL}$ of $\mathrm{HF}$, both sub-boiling distillates. The material was heated to $200^{\circ} \mathrm{C}$ in an electric oven, under pressure, for five days. The material was then cooled and transferred to a PFA beaker where the solution was heated in hot plate; in this process, $\mathrm{SiF}_{4}$ is eliminated by evaporation. After drying, the material is dissolved and transferred to a plastic bottle where weights were adjusted to $100 \mathrm{~g}$ with $1 \% \mathrm{HNO}_{3}$. Analytical quality control was checked by the analyses of reference materials JG-1a and JR-1 as unknown, blank and duplicate samples. The values obtained should be within $2 \sigma$ of the certified values, and the duplicated samples within $1 \%$ of variation (Navarro et al. 2008).

\section{Zircon concentration, mounting and cathodoluminescence study}

The separation and concentration of zircon consisted of crushing, grinding, sieving, vibrating table, electromagnetic separation, heavy liquids and finally manual picking.
The routine used at CPGeo is described by Sato et al. (2014) and consists of the following steps:

rock samples $(0.5$ to $3 \mathrm{~kg}$ for granites and - $20 \mathrm{~kg}$ for mafic rocks) are crushed in a jaw crusher;

- crushed material is screened to separate a fraction of 100-250 mesh particle size, using a disk mill and a battery of sieves;

the separated fraction is taken to a vibratory table to concentrate heavy minerals;

- magnetic minerals are removed with a hand magnet;

- minerals with different magnetic susceptibilities are concentrated in a Frantz-type magnetic separator, by varying the inclination and the intensity of the electromagnetic field;

the least magnetic fractions are passed successively into bromoform $\left(\mathrm{d}=2.85 \mathrm{~g} / \mathrm{cm}^{3}\right)$ and methylene iodide $\left(\mathrm{d}=3.2 \mathrm{~g} / \mathrm{cm}^{3}\right)$ to further concentrate the heavy minerals of interest. Any remaining sulfides present in the concentrates are eliminated with $\mathrm{HCl}$ or $\mathrm{HNO}_{3}$. About 50-100 zircon grains are separated by hand picking with the aid of a magnifying microscope, and then mounted on double sided adhesive tape and embedded in epoxy-type resin. The mount is then polished to expose the fresh surface of the grain trapped in the resin.

A previous study of cathodoluminescence (CL) images of each of the samples was necessary to choose the proper position of the spot during the pointed analyses by SHRIMP. After a thin layer of gold cover $(2-3 \mathrm{~nm})$ was added to the mounts, these images were obtained in a FEI Quanta 250 SEM spectrometer with a XMAX CL detector (Oxford Instruments). Operating conditions were: high voltage, $15 \mathrm{kV}$, distance, 16.9-17 mm, PMD detector, range of magnification, 95-250x.

\section{$\mathrm{U}-\mathrm{Pb}$ geochronology by SHRIMP}

$\mathrm{U}-\mathrm{Pb}$ zircon dating was performed in the SHRIMP IIe equipment installed at CPGeo, Instituto de Geociências, Universidade de São Paulo. The SHRIMP is a mass spectrometer coupled to a high resolution ion microprobe that uses a collimated and accelerated beam of primary ions (O2) to reach a target where secondary ions are generated from $30-\mathrm{m}$ spots. The secondary ions are accelerated through the equipment and the isotopes generated by the sample, ${ }^{254} \mathrm{UO}+$, ${ }^{206} \mathrm{~Pb}+{ }^{207} \mathrm{~Pb}+,{ }^{208} \mathrm{~Pb}+,{ }^{238} \mathrm{U}+$ and $+{ }^{254} \mathrm{ThO}$ and ${ }^{196} \mathrm{Zr} 2 \mathrm{O}+$ are measured successively. The corrections required by the technique are made by the analyses of unknown materials and reference materials with known isotopic ratios (matrix-matched) for determining specific calibration factors (Black et al. 2004). 12-16 spot analyses from each of the unknown zircon samples are performed according to the method described 
in Sato et al. (2014). During the run of every 5 determinations analyzed, the Temora-2 reference material (estimated age $416.78 \pm 0.33 \mathrm{Ma}$; Black et al. 2004) was used as ${ }^{206} \mathrm{~Pb} /{ }^{238} \mathrm{U}$ age reference, for calculation of common $\mathrm{Pb}$ correction factors and fractionation factors. Common lead corrections usually use ${ }^{204} \mathrm{~Pb}$ according to Stacey and Kramer (1975). Reference material SL13 (238 ppm) is used as U composition reference. Data are reduced with SQUID 1.6 software (Ludwig 2009) and ISOPLOT 4 (Ludwig 2003) has been used for treatment of data to estimate ages and generate diagrams.

\section{U-Pb ZIRCON DATING}

\section{Porphyritic hornblende-biotite granite (Bragança Paulista-type, HKCA)}

\section{Sample petrography and geochemistry}

The porphyritic hornblende-biotite granites of HKCA character correspond to the Bragança Paulista association, which are the most voluminous rocks in the Socorro Batholith. We chose two samples from localities in the northern and southern portion of the batholith for U-Pb dating (Fig. 2), representing compositions with different degrees of fractionation.

Sample BRP-08 is from the vicinities of the Pedra Bela hill, and corresponds to a porphyritic hornblende-biotite granite with high color index $(-22)$, with abundant and very large K-feldspar megacrysts averaging 3-4 × 1.5-2 cm (Fig. 3A) set in a massive, coarse matrix where plagioclase occurs as the only feldspar.

Sample ATB-13 is from a road next to the SP-095, NW of Bragança Paulista; compared to BRP-08, this sample has lower color index $(-15)$, and the K-feldspar megacrysts are a little smaller and more elongated (average 2-3 $21 \mathrm{~cm}$ ); a slight solid-state foliation is evident, which is related to a tensional field and evidenced by the orientation of alkali feldspars.

Our geochemical dataset of the Bragança Paulista type granites (Tabs. 1 and 2) shows that they are relatively primitive, with 60-66 wt $\% \mathrm{SiO}_{2}, \mathrm{mg \#}-40$, combining relatively high contents of $\mathrm{MgO}(2.5-1.5 \mathrm{wt} \%), \mathrm{CaO}(4.5-3.2 \mathrm{wt} \%)$ and $\mathrm{Fe}_{2} \mathrm{O}_{3}(6.2-4.3 \mathrm{wt} \%)$ with high $\mathrm{K}_{2} \mathrm{O}(3.8-4.6 \mathrm{wt} \%)$, $\mathrm{Ba}(1,000-1,600 \mathrm{ppm})$ and $\mathrm{Sr}(540-800 \mathrm{ppm})$. BRP-08 with $61.7 \mathrm{wt} \% \mathrm{SiO}_{2}$ and $2.0 \mathrm{wt} \% \mathrm{MgO}$ is among the most unfractioned compositions, while ATI-13 with 66.3 wt $\%$ $\mathrm{SiO}_{2}$ and $1.5 \mathrm{wt} \% \mathrm{MgO}$ groups with the most felsic Bragança Paulista-type granites (Fig. 4). Both samples have moderately fractionated REE patterns $(\mathrm{La} / \mathrm{Yb})_{\mathrm{N}}=24-30$ and discreet negative Eu anomalies (Fig. 5), that are more pronounced in the least fractioned sample $\left(\mathrm{BRP}=08 ; \mathrm{Eu}_{\mathrm{N}} / \mathrm{Eu}^{*}=0.70\right.$, versus 0.83 in ATB-13 $\left(\mathrm{Eu}^{*}=(\mathrm{Sm} x \mathrm{Gd}) 1 / 2\right)$.

\section{Zircon morphology and U-Pb dating}

Zircon crystals from sample BRP-08 are elongated with aspect ratios from $4: 1$ to $5: 1$, and lengths up to $400 \mu \mathrm{m}$ (Suppl. data). CL images show typical oscillatory zoning, which is more pronounced in darker zones where small inclusions that are white in CL are present. Lighter cores with less evident zoning and a thin CL-bright outer rim, sometimes truncating the oscillatory zoning, occur in some crystals.

SHRIMP results from 21 spots yield essentially concordant ages (typical discordance $\leq 4 \%$; Tab. 3 ) which, however, spread over the concordia for ca. 50 M.y. (584-635 Ma, excluding three extreme results: the two youngest and the oldest one). The weighted average ${ }^{206} \mathrm{~Pb} /{ }^{238} \mathrm{U}$ age (Fig. 6) calculated by Isoplot is $610.1 \pm 7.0 \mathrm{Ma}$ (at $95 \%$ confidence), with a very high MSDW (6.1). Use of the Isoplot Unmix routine discriminates two age groups with $600.7 \pm 3.9 \mathrm{Ma}$ (eight samples) and $623.4 \pm 4.8 \mathrm{Ma}$ (six samples) (errors in calculated ages are reported as $2 \sigma)$. A concordia age calculated
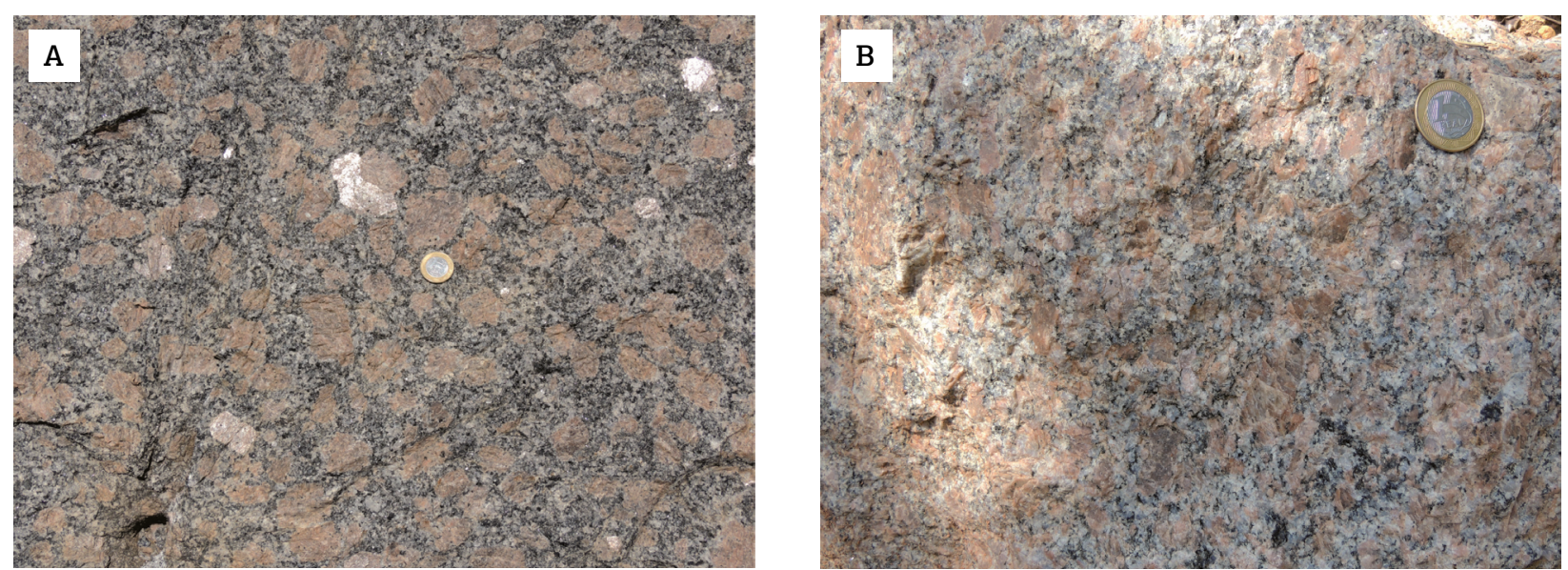

Figure 3. (A) Image of Bragança Paulista-type from the Pedra Bela hill. (B) Image of the dated sample of Salmãotype granite. 
Table 1. Major and trace elements of granites from the Socorro Batholith by X-ray fluorescence.

\begin{tabular}{|c|c|c|c|c|c|c|c|c|c|c|c|c|c|c|c|}
\hline & \multicolumn{2}{|c|}{$\begin{array}{l}\text { Salmão } \\
\text { Granite }\end{array}$} & \multicolumn{8}{|c|}{ Bragança Paulista Granite } & \multicolumn{2}{|c|}{$\begin{array}{c}\text { Socorro } \\
\text { Charnockite }\end{array}$} & \multicolumn{3}{|c|}{ Atibaia Charnockite } \\
\hline & $\begin{array}{l}\text { BRP- } \\
03\end{array}$ & $\begin{array}{l}\text { BRP- } \\
\text { 06C }\end{array}$ & $\begin{array}{c}\text { BRP- } \\
13\end{array}$ & $\begin{array}{l}\text { BRP- } \\
\text { 06A }\end{array}$ & $\begin{array}{l}\text { BRP- } \\
\text { 06B }\end{array}$ & $\begin{array}{c}\text { BRP- } \\
07\end{array}$ & $\begin{array}{c}\text { BRP- } \\
08\end{array}$ & $\begin{array}{c}\text { BRP- } \\
09\end{array}$ & $\begin{array}{c}\text { BRP- } \\
10\end{array}$ & $\begin{array}{c}\text { ATB- } \\
13\end{array}$ & $\begin{array}{c}\text { BRP- } \\
11\end{array}$ & $\begin{array}{c}\text { BRP- } \\
12\end{array}$ & $\begin{array}{l}\text { ATB- } \\
06 \mathrm{~A}\end{array}$ & $\begin{array}{l}\text { ATB- } \\
08\end{array}$ & $\begin{array}{l}\text { BRP- } \\
05\end{array}$ \\
\hline $\mathrm{SiO}_{2}$ & 3.55 & 72.81 & 63.89 & 60.69 & 64.77 & 64.59 & 61.71 & 63.58 & 65.95 & 66.30 & 63.75 & 61.68 & 69.10 & 69.77 & 68.47 \\
\hline $\mathrm{TiO}_{2}$ & 0.22 & 0.45 & 0.87 & 1.18 & 0.862 & 0.93 & 1.15 & 1.17 & 0.89 & 0.80 & 1.15 & 0.92 & 0.53 & 0.46 & 0.54 \\
\hline $\mathrm{Al}_{2} \mathrm{O}_{3}$ & 13.34 & 13.30 & 15.23 & 15.77 & 14.63 & 14.74 & 15.15 & 14.85 & 14.74 & 14.61 & 14.92 & 16.22 & 14.46 & 14.66 & 14.23 \\
\hline $\mathrm{Fe}_{2} \mathrm{O}_{3}$ & 1.29 & 2.27 & 4.47 & 5.99 & 4.42 & 4.60 & 5.71 & 6.24 & 4.47 & 4.29 & 5.90 & 5.62 & 3.76 & 3.04 & 3.62 \\
\hline $\mathrm{MnO}$ & 0.02 & 0.02 & 0.07 & 0.09 & 0.058 & 0.07 & 0.08 & 0.10 & 0.07 & 0.08 & 0.10 & 0.11 & 0.05 & 0.03 & 0.03 \\
\hline $\mathrm{MgO}$ & 0.29 & 0.58 & 1.66 & 2.23 & 1.59 & 1.65 & 2.02 & 2.04 & 1.50 & 1.55 & 2.01 & 2.19 & 0.62 & 0.57 & 0.66 \\
\hline $\mathrm{CaO}$ & 1.45 & 1.79 & 3.57 & 4.58 & 3.50 & 3.71 & 3.91 & 4.22 & 3.59 & 3.18 & 4.06 & 4.56 & 1.91 & 2.03 & 1.85 \\
\hline $\mathrm{Na}_{2} \mathrm{O}$ & 2.81 & 2.32 & 3.09 & 3.19 & 2.82 & 2.64 & 3.10 & 2.94 & 2.95 & 2.94 & 2.97 & 3.09 & 2.79 & 2.81 & 2.83 \\
\hline $\mathrm{K}_{2} \mathrm{O}$ & 5.22 & 5.39 & 4.59 & 4.08 & 4.80 & 4.54 & 4.63 & 3.58 & 4.44 & 4.70 & 3.67 & 3.76 & 5.76 & 5.88 & 5.73 \\
\hline $\mathrm{P}_{2} \mathrm{O}_{5}$ & 0.04 & 0.11 & 0.33 & 0.39 & 0.327 & 0.31 & 0.37 & 0.41 & 0.30 & 0.30 & 0.39 & 0.35 & 0.15 & 0.15 & 0.15 \\
\hline LoI & 0.46 & 0.72 & 0.78 & 1.98 & 1.59 & 0.78 & 0.81 & 0.80 & 0.74 & 0.29 & 0.75 & 0.66 & 0.32 & 0.32 & 0.53 \\
\hline $\begin{array}{l}\text { Total } \\
(\%)\end{array}$ & 8.68 & 99.76 & 98.56 & 100.16 & 99.37 & 98.55 & 98.64 & 99.93 & 99.64 & 99.029 & 99.67 & 99.16 & 99.45 & 99.722 & 98.63 \\
\hline $\mathrm{Ba}$ & 541 & 1397 & 1350 & 1.600 & 1622 & 1581 & 1584 & 1.058 & 1290 & 1.340 & 1076 & 8 & 76 & 654 & 651 \\
\hline $\mathrm{Ce}$ & 134 & 231 & 122 & 169 & 156 & 127 & 189 & 190 & 107 & 120 & 199 & 127 & 186 & 168 & 230 \\
\hline Co & $<6$ & $<6$ & 7 & 12 & 7 & 8 & 10 & 9 & 6 & 6 & 8 & 9 & $<6$ & $<6$ & $<6$ \\
\hline $\mathrm{Cr}$ & $<13$ & $<13$ & 20 & 24 & 16 & 16 & 20 & $<13$ & $<13$ & 20 & $<13$ & 13 & $<13$ & $<13$ & $<13$ \\
\hline $\mathrm{Cu}$ & $<5$ & 7 & 9 & 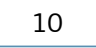 & 15 & 8 & 12 & 10 & 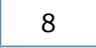 & 9 & 10 & 14 & 6 & 6 & 5 \\
\hline $\mathrm{Ga}$ & 19 & 15 & 23 & 23 & 19 & 21 & 21 & 21 & 19 & 20 & 23 & 20 & 19 & 18 & 20 \\
\hline $\mathrm{La}$ & 73 & 124 & 61 & 90 & 82 & 60 & 88 & 103 & 66 & 72 & 101 & 70 & 88 & 91 & 121 \\
\hline $\mathrm{Nb}$ & $<9$ & $<9$ & 18 & 14 & 11 & 17 & 20 & 19 & 13 & 15 & 18 & 14 & 13 & 12 & 11 \\
\hline $\mathrm{Nd}$ & 41 & 57 & 50 & 62 & 51 & 60 & 75 & 67 & 40 & 37 & 73 & 49 & 58 & 53 & 73 \\
\hline $\mathrm{Ni}$ & $<5$ & $<5$ & 11 & 10 & 9 & 9 & 9 & 8 & 7 & 11 & 8 & 11 & $<5$ & $<5$ & $<5$ \\
\hline $\mathrm{Pb}$ & 30 & 21 & 19 & 18 & 13 & 16 & 19 & 15 & 18 & 20 & 14 & 20 & 18 & 25 & 17 \\
\hline $\mathrm{Rb}$ & 183 & 118 & 153 & 126 & 134 & 135 & 153 & 126 & 131 & 157 & 130 & 111 & 166 & 168 & 176 \\
\hline $\mathrm{Sc}$ & $<14$ & $<14$ & $<14$ & 14 & $<14$ & $<14$ & 14 & 14 & $<14$ & $<14$ & 15 & 16 & $<14$ & $<14$ & $<14$ \\
\hline $\mathrm{Sr}$ & 183 & 643 & 681 & 783 & 748 & 678 & 658 & 542 & 626 & 647 & 544 & 799 & 141 & 170 & 162 \\
\hline Th & 35 & 31 & $<7$ & 18 & 10 & $<7$ & 7 & 13 & 7 & 10 & 13 & 0 & 14 & 17 & 30 \\
\hline $\mathrm{U}$ & 4 & 7 & 8 & 10 & 8 & 8 & 9 & 9 & 7 & 9 & 8 & 8 & 5 & 6 & 5 \\
\hline $\mathrm{V}$ & 10 & 36 & 74 & 100 & 74 & 80 & 99 & 96 & 71 & 65 & 84 & 102 & 29 & 21 & 31 \\
\hline $\mathrm{Y}$ & 5 & 8 & 28 & 21 & 17 & 28 & 31 & 32 & 19 & 18 & 29 & 26 & 16 & 11 & 9 \\
\hline $\mathrm{Zn}$ & 22 & 30 & 65 & 90 & 69 & 73 & 89 & 90 & 67 & 62 & 86 & 74 & 50 & 41 & 54 \\
\hline $\mathrm{Zr}$ & 159 & 197 & 250 & 271 & 254 & 261 & 318 & 341 & 223 & 252 & 328 & 231 & 415 & 402 & 397 \\
\hline
\end{tabular}

Table 2. Rare earth and additional trace elements of granites from the Socorro Batholith by ICPMS.

\begin{tabular}{|c|c|c|c|c|c|c|c|c|c|c|c|c|c|c|c|c|c|c|}
\hline Sample & La & $\mathrm{Ce}$ & $\operatorname{Pr}$ & Nd & $\mathrm{Sm}$ & $\mathbf{E u}$ & Gd & $\mathrm{Tb}$ & Dy & Ho & Er & $\mathrm{Tm}$ & $\mathrm{Yb}$ & Lu & Hf & $\mathrm{Pb}$ & Th & $\mathbf{U}$ \\
\hline BRP-03 & 75.83 & 134.69 & 14.23 & 44.21 & 6.25 & 1.13 & 2.75 & 0.33 & 1.24 & 0.18 & 0.39 & 0.05 & 0.31 & 0.05 & 4.70 & 33.05 & 36.17 & 1.56 \\
\hline BRP-08 & 86.71 & 174.14 & 20.68 & 76.57 & 12.59 & 2.31 & 8.16 & 1.06 & 5.58 & 1.05 & 2.75 & 0.39 & 2.39 & 0.33 & 8.45 & 17.44 & 5.70 & 0.89 \\
\hline BRP-12 & 65.47 & 129.80 & 14.32 & 52.26 & 9.04 & 2.13 & 5.84 & 0.83 & 4.52 & 0.85 & 2.24 & 0.33 & 2.04 & 0.29 & 5.40 & 17.08 & 6.74 & 0.42 \\
\hline ATB-08 & 84.28 & 160.27 & 17.37 & 56.65 & 8.39 & 1.34 & 4.09 & 0.56 & 2.45 & 0.42 & 1.01 & 0.12 & 0.69 & 0.10 & 8.73 & 20.61 & 14.31 & 0.71 \\
\hline ATB-13 & 65.27 & 120.23 & 13.26 & 45.41 & 7.85 & 1.76 & 5.18 & 0.71 & 3.50 & 0.64 & 1.62 & 0.23 & 1.36 & 0.20 & 6.80 & 18.64 & 9.08 & 0.89 \\
\hline
\end{tabular}

ICPMS: inductively coupled plasma mass spectrometry. 

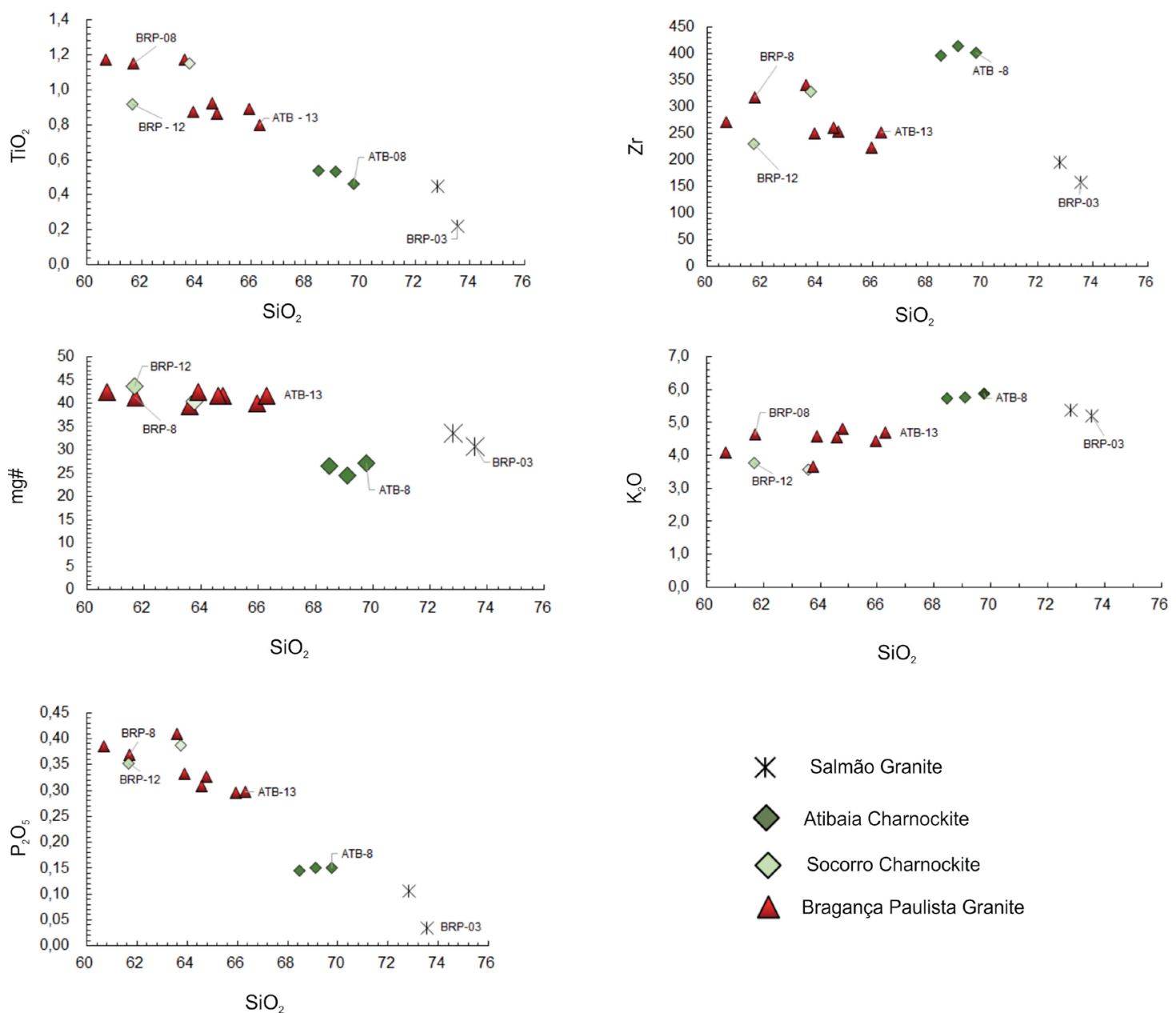

Figure 4. Geochemical variation diagrams for samples analyzed in this study and related samples.

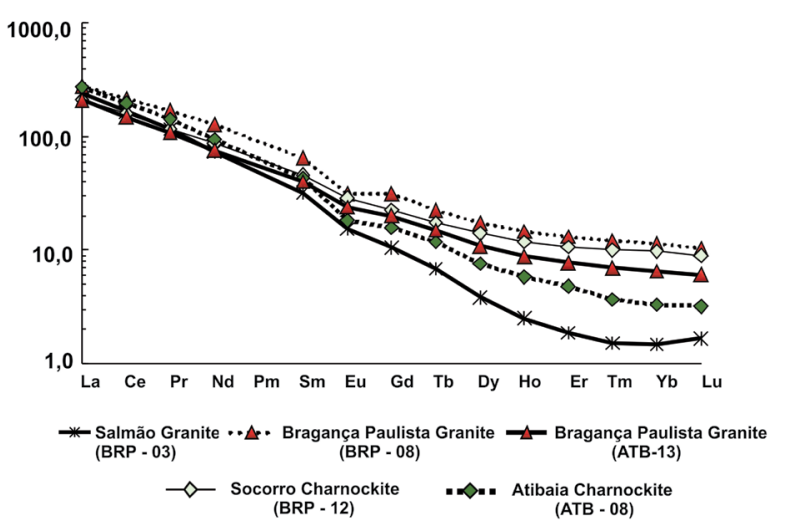

Figure 5. Chondrite-normalized (Boynton, 1984) REE patterns of dated samples.

for the first set yields $599.5 \pm 3.6$ (MSWD $=0.25$ ) with a probability of fit of 0.62 , while the concordia age for the second set is $620.1 \pm 4.2 \mathrm{Ma}$, with higher MSWD (0.94) and lower probability of fit (0.33). No morphological or chemical differences exist between spots belonging to these two age groups, and some of the "older" dates correspond to spots located at the border of zircon crystals. We prefer therefore to admit the weighted average age of the whole set of analyses as the best estimate of the magmatic crystallization of this sample, in spite of the higher associated MSWD. Indeed, the Unmix routine can yield any chosen number of populations, but in this case no clear age gap is observed when the whole set of data is considered and no chemical or textural contrast exists between the different spots.

Zircons from sample ATB-13 have varied morphologies, with wide variation in aspect ratios, from $1.5: 1$ to $4: 1$ (Suppl. data). Most crystals are elongated, with lengths of $100-400 \mu \mathrm{m}$, and show oscillatory zoning, small granular inclusions of a CL-bright mineral, possibly apatite, occurring associated with some specific zones, and rare brighter cores. The shorter crystals show similar features, and more commonly have corroded cores of varied texture; one CL-bright homogeneous core corresponds to an inherited crystal.

Fourteen spots were analyzed, and yield nearly concordant ages ( $\leq 5 \%$ discordant, with two exceptions, Tab. 3 ). Spot 13.1 is an inherited core with a concordant ${ }^{207} \mathrm{~Pb} /{ }^{206} \mathrm{~Pb}$ age of 1,445 


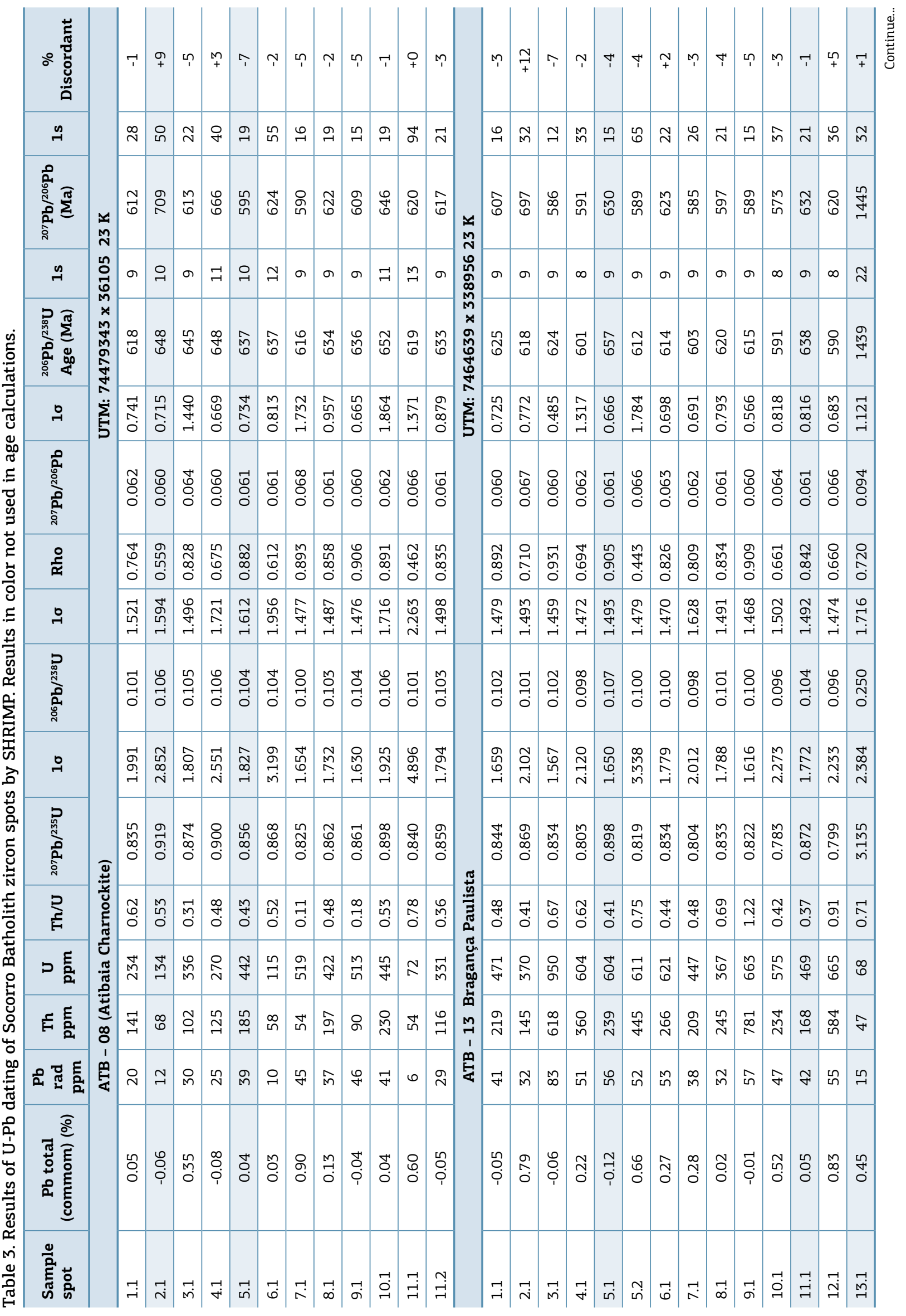




\begin{tabular}{|c|c|c|c|c|c|c|c|c|c|c|c|c|c|c|c|c|c|c|c|c|c|c|c|c|c|c|c|}
\hline 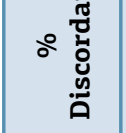 & & $\not r$ & -1 & $-r$ & $M$ & 0 & m & $\approx$ & $\approx$ & $F^{\prime}$ & Э & $r$ & $N$ & ન & $\tilde{\sigma}$ & $\vec{T}$ & $\tilde{N}$ & & $m$ & $M$ & $\sim$ & ب & $m$ & $\nabla$ & $?$ & $F^{\prime}$ & $m$ \\
\hline 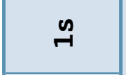 & & $\approx$ & $\vec{N}$ & $\underset{7}{\ni}$ & $\stackrel{\infty}{\sim}$ & $\exists$ & m & F & 芯 & ฟ & $\stackrel{\sim}{\sim}$ & 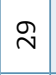 & $\vec{\lambda}$ & $\stackrel{\curvearrowleft}{\sim}$ & m & $\stackrel{\infty}{\infty}$ & $\stackrel{M}{\sim}$ & & $\widehat{\sim}$ & $\widehat{\sim}$ & $\stackrel{\circ}{\stackrel{2}{1}}$ & M & $\underset{f}{y} \approx$ & ஸే & $\stackrel{n}{\sim}$ & 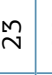 & $\stackrel{\nu}{N}$ \\
\hline 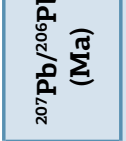 & $\begin{array}{l}\mathbf{y} \\
\boldsymbol{N} \\
\boldsymbol{N}\end{array}$ & 尺ু & $\widehat{\widehat{\sigma}}$ & ஸ̂̃ & $\begin{array}{l}0 \\
\vec{\sigma}\end{array}$ & $\stackrel{\infty}{\widetilde{U}}$ & ڤิ & $\begin{array}{l}\hat{\tilde{\omega}} \\
\hat{\sigma}\end{array}$ & 苫 & mo & 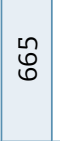 & $\begin{array}{l}\infty \\
\substack{0 \\
0}\end{array}$ & $\underset{\hat{A}}{\stackrel{\hat{\sigma}}{*}}$ & $\begin{array}{l}n \\
\widetilde{\sigma}\end{array}$ & $\begin{array}{l}0 \\
\text { ôn } \\
\end{array}$ & \begin{tabular}{|l|}
$\infty$ \\
$\overrightarrow{6}$
\end{tabular} & $\stackrel{M}{N}$ & $\begin{array}{l}x \\
n \\
N\end{array}$ & 芯 & $\vec{b}$ & 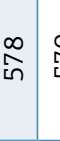 & 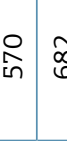 & 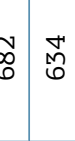 & న్ & กิ & 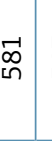 & 总 \\
\hline$\stackrel{n}{\rightarrow}$ & 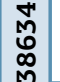 & in & in & in & in & in & in & $\stackrel{\Re}{N}$ & $\stackrel{M}{\rightarrow}$ & in & in & in & $\stackrel{\infty}{\sim}$ & $r$ & $a$ & 0 & $\bullet$ & 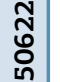 & $\stackrel{m}{n}$ & ஸึ & 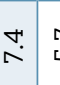 & $\begin{array}{ccc}\hat{i n} & \infty \\
\end{array}$ & $\begin{array}{lll}\infty & 0 \\
\end{array}$ & Mn & हैं & ஸn & $\stackrel{\circ}{\circ}$ \\
\hline 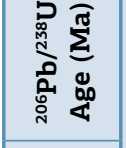 & 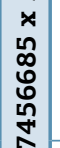 & సู & $\widetilde{\widetilde{U}}$ & ָิ & $\hat{\tilde{N}}$ & O̊ & $\vec{\sigma}$ & 芯 & $\begin{array}{l}0 \\
6 \\
0\end{array}$ & 甪 & 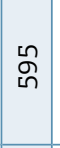 & $\overrightarrow{\widehat{C}}$ & 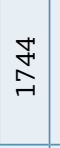 & $\begin{array}{l}\infty \\
\ddots 1 \\
n\end{array}$ & $\hat{\tilde{N}}$ & fิ & ठ্ট & $\begin{array}{l}m \\
x \\
n \\
\hat{n} \\
\hat{\alpha} \\
\alpha \\
y\end{array}$ & $\begin{array}{lll}n & 5 \\
\infty & 0 \\
\infty & \vdots \\
n & \vdots\end{array}$ & 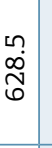 & $\begin{array}{c}0 \\
\infty \\
o \\
\vdots \\
n\end{array}$ & & 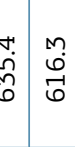 & $\begin{array}{l}\infty \\
0 \\
0 \\
0 \\
i n\end{array}$ & 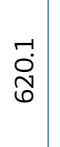 & 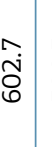 & 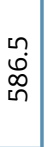 \\
\hline 욤 & $\sum_{\dot{S}}^{\ddot{s}}$ & $\begin{array}{l}0 \\
0 \\
0 \\
0 \\
\end{array}$ & $\begin{array}{c}N \\
\hat{O} \\
0\end{array}$ & 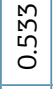 & $\begin{array}{c}0 \\
M \\
\infty \\
0 \\
0\end{array}$ & 离 & 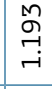 & $\begin{array}{c}M \\
m \\
\infty \\
-i \\
-i\end{array}$ & 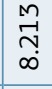 & $\begin{array}{l}n \\
0 \\
0 \\
0 \\
0\end{array}$ & $\begin{array}{l}\infty \\
\infty \\
o \\
0 \\
0\end{array}$ & $\begin{array}{c}\vec{M} \\
\stackrel{-}{r} \\
-\end{array}$ & 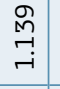 & $\begin{array}{l}n \\
0 \\
m \\
0 \\
0\end{array}$ & $\begin{array}{l}\vec{D} \\
\dot{i} \\
\end{array}$ & 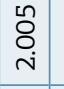 & $\begin{array}{l}\overrightarrow{0} \\
\text { Oे } \\
\end{array}$ & $\sum_{j}^{\ddot{z}}$ & 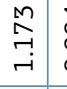 & 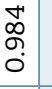 & 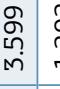 & 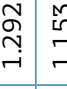 & 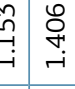 & 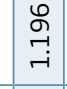 & $\begin{array}{l}\tilde{0} \\
0 \\
0 \\
0\end{array}$ & $\begin{array}{l}0 \\
ِ \\
-i \\
\end{array}$ & 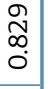 \\
\hline 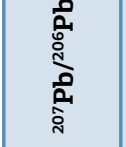 & & $\begin{array}{l}\text { o } \\
\text { o. }\end{array}$ & $\begin{array}{l}\vec{b} \\
0 \\
0\end{array}$ & $\begin{array}{l}-1 \\
0 \\
0 \\
0\end{array}$ & $\begin{array}{l}\not \\
0 \\
0\end{array}$ & $\vec{o}$ & $\begin{array}{l}\vec{~} \\
\stackrel{0}{0} \\
0\end{array}$ & $\begin{array}{l}\tilde{N} \\
\stackrel{0}{G} \\
0\end{array}$ & $\begin{array}{l}\vec{z} \\
\overrightarrow{1} \\
0\end{array}$ & $\begin{array}{l}\text { ô } \\
\text { ô. } \\
\text { o. }\end{array}$ & $\begin{array}{l}\tilde{O} \\
0 \\
0 \\
0\end{array}$ & $\begin{array}{l}\overrightarrow{0} \\
0 \\
0\end{array}$ & $\begin{array}{l}0 \\
\text { Oे } \\
\text { - }\end{array}$ & $\begin{array}{l}0 \\
\delta \\
0 \\
0\end{array}$ & $\begin{array}{l}\hat{o} \\
\text { Oे } \\
0\end{array}$ & $\begin{array}{l}n \\
0 \\
0 \\
0\end{array}$ & $\begin{array}{l}\vec{\Xi} \\
\stackrel{0}{0} \\
0\end{array}$ & & $\begin{array}{l}\vec{\Xi} \\
0 \\
0\end{array}$ & $\begin{array}{l}\overrightarrow{8} \\
0 \\
0\end{array}$ & $\begin{array}{c}n \\
\vdots \\
0 \\
0\end{array}$ & 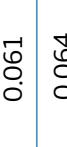 & 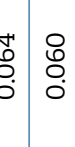 & $\begin{array}{l}\tilde{\delta} \\
\stackrel{0}{0} \\
0\end{array}$ & $\begin{array}{l}0 \\
0 \\
0 \\
0\end{array}$ & 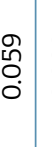 & $\begin{array}{l}0 \\
\varnothing \\
0\end{array}$ \\
\hline 总 & & $\begin{array}{l}\hat{n} \\
\hat{0}\end{array}$ & $\begin{array}{l}-\vec{\infty} \\
0 \\
0 \\
0\end{array}$ & $\begin{array}{l}-1 \\
0 \\
0 \\
0\end{array}$ & $\begin{array}{l}0 \\
\mathfrak{n} \\
\hat{o} \\
0\end{array}$ & $\begin{array}{c}m \\
n \\
\infty \\
0 \\
0\end{array}$ & $\begin{array}{l}ت \\
\vec{H} \\
0\end{array}$ & 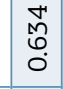 & $\begin{array}{l}M \\
0 \\
0 \\
0 \\
0\end{array}$ & $\begin{array}{l}\hat{n} \\
\hat{0} \\
0\end{array}$ & $\begin{array}{l}n \\
\hat{0} \\
0 \\
0\end{array}$ & \begin{tabular}{l}
$\infty$ \\
\multirow{1}{*}{} \\
\\
0
\end{tabular} & $\begin{array}{c}\infty \\
m \\
\infty \\
0 \\
0\end{array}$ & $\begin{array}{c}n \\
\hat{\alpha} \\
0 \\
0\end{array}$ & $\begin{array}{l}\infty \\
\overrightarrow{7} \\
0 \\
0\end{array}$ & $\begin{array}{l}\text { స్ } \\
\text { Oे } \\
\end{array}$ & $\begin{array}{l}\hat{\hat{o}} \\
\stackrel{0}{0}\end{array}$ & & \begin{tabular}{l|l}
0 \\
0 \\
0 \\
0
\end{tabular} & 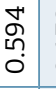 & 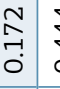 & 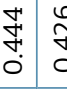 & 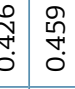 & $\begin{array}{l}\tilde{H} \\
\stackrel{0}{0} \\
0\end{array}$ & $\begin{array}{l}0 \\
\infty \\
0 \\
0\end{array}$ & $\begin{array}{l}N \\
\hat{b} \\
0 \\
0\end{array}$ & $\begin{array}{l}+1 \\
0 \\
0 \\
0\end{array}$ \\
\hline 움 & & $\begin{array}{c}\mathcal{N} \\
\infty \\
0 \\
0\end{array}$ & $\begin{array}{l}0 \\
0 \\
\infty \\
0 \\
0\end{array}$ & $\begin{array}{l}-\overrightarrow{1} \\
\vec{\sigma} \\
0\end{array}$ & $\begin{array}{l}8 \\
8 \\
0 \\
0\end{array}$ & $\begin{array}{l}n \\
\vdots \\
\infty \\
0 \\
0\end{array}$ & $\begin{array}{l}\hat{D} \\
\infty \\
0 \\
0\end{array}$ & $\begin{array}{l}M \\
\stackrel{n}{i} \\
\stackrel{i}{i}\end{array}$ & 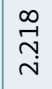 & \begin{tabular}{|c|}
$n$ \\
0 \\
$\infty$ \\
0 \\
0
\end{tabular} & $\begin{array}{l}0 \\
\infty \\
\infty \\
0\end{array}$ & $\begin{array}{l}M \\
\infty \\
\infty \\
0\end{array}$ & $\begin{array}{l}\infty \\
\infty \\
\infty \\
-i\end{array}$ & $\begin{array}{l}M \\
N \\
\infty \\
0\end{array}$ & $\begin{array}{c}\infty \\
\stackrel{1}{1} \\
-i\end{array}$ & \begin{tabular}{|l|}
0 \\
$\circ$ \\
$\circ$ \\
0 \\
\end{tabular} & $\begin{array}{l}0 \\
\text { ஸू } \\
0 \\
0\end{array}$ & & 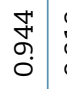 & $\begin{array}{l}\sigma \\
\tilde{\sigma} \\
\sigma\end{array}$ & \begin{tabular}{c|c}
$\hat{o}$ & $\vdots$ \\
$\omega$ & $\vdots$ \\
$-i$ &
\end{tabular} & \begin{tabular}{l|l} 
& 0 \\
0 & 0 \\
0 & $b$ \\
0 & 0 \\
$c$
\end{tabular} & 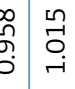 & 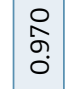 & $\begin{array}{l}0 \\
\text { ô } \\
0 \\
0 \\
0\end{array}$ & $\begin{array}{c}n \\
\text { ñ } \\
\text { ô } \\
\text { d }\end{array}$ & $\begin{array}{l}\mathscr{\sigma} \\
\infty \\
0 \\
0\end{array}$ \\
\hline 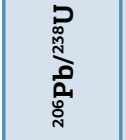 & & \begin{tabular}{l}
$\overrightarrow{0}$ \\
\multirow{1}{0}{} \\
0
\end{tabular} & $\begin{array}{l}\overrightarrow{0} \\
\overrightarrow{1} \\
0\end{array}$ & $\mid \begin{array}{c}\overrightarrow{0} \\
\stackrel{1}{0}\end{array}$ & 誉 & $\begin{array}{l}\text { mo } \\
\stackrel{9}{a} \\
0\end{array}$ & 总 & 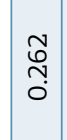 & 8) & $\begin{array}{l}\text { mo } \\
\stackrel{1}{0}\end{array}$ & $\begin{array}{l}\hat{o} \\
0 \\
0\end{array}$ & $\begin{array}{l}\overrightarrow{0} \\
-1 \\
0 \\
0\end{array}$ & $\begin{array}{c}\overrightarrow{1} \\
\bar{n} \\
0\end{array}$ & $\begin{array}{l}\stackrel{\Xi}{0} \\
0 \\
0 \\
0\end{array}$ & $\begin{array}{l}n \\
\hat{n} \\
0 \\
0\end{array}$ & $\begin{array}{l}0 \\
\stackrel{0}{0} \\
0 \\
0\end{array}$ & $\begin{array}{l}\infty \\
\circ \\
0 \\
0\end{array}$ & & $\begin{array}{l}n \\
\text { Oे. } \\
0\end{array}$ & 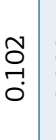 & $\begin{array}{l}\tilde{O} \\
\tilde{O} \\
0 \\
0\end{array}$ & 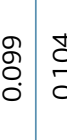 & \begin{tabular}{l|l}
0 \\
\\
\end{tabular} & $\begin{array}{l}\text { ô } \\
\text { Oे }\end{array}$ & $\begin{array}{l}\overrightarrow{0} \\
\text { ज्ञ } \\
\text {. }\end{array}$ & ס & $\begin{array}{l}n \\
0 \\
0 \\
0\end{array}$ \\
\hline 움 & & $\begin{array}{l}\vec{b} \\
\overrightarrow{-} \\
-1\end{array}$ & $\begin{array}{c}0 \\
0 \\
m \\
\rightarrow-1\end{array}$ & $\mid \begin{array}{c}\infty \\
0 \\
0 \\
-i \\
-i\end{array}$ & ָָ & $\begin{array}{l}\text { Oे } \\
\text { o. } \\
\text { Oे }\end{array}$ & $\underset{\substack{\infty \\
\rightarrow \\
\sim}}{\sim}$ & $\begin{array}{l}\hat{y} \\
\dot{y} \\
\dot{n}\end{array}$ & $\begin{array}{l}\stackrel{\rho}{a} \\
\infty \\
\dot{\omega} \\
\dot{\sim}\end{array}$ & 品 & $\begin{array}{c}\mathcal{N} \\
\stackrel{m}{r} \\
-\end{array}$ & 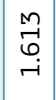 & $\underset{\hat{n}}{\stackrel{n}{i}}$ & $\begin{array}{l}n \\
\hat{o} \\
r i\end{array}$ & 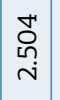 & $\begin{array}{l}\overrightarrow{\mid} \\
\stackrel{8}{m} \\
\text { in }\end{array}$ & 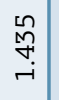 & & 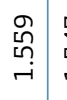 & $\begin{array}{c}\hat{f} \\
\text { ț } \\
\end{array}$ & 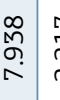 & 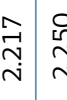 & 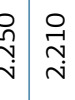 & $\begin{array}{l}\hat{o} \\
\dot{\omega} \\
i\end{array}$ & $\begin{array}{l}\stackrel{H}{0} \\
\stackrel{-}{i} \\
\end{array}$ & $\overrightarrow{\vec{F}}$ & $\begin{array}{l}\stackrel{\bigcirc}{9} \\
\stackrel{-}{-}\end{array}$ \\
\hline 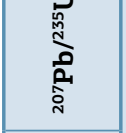 & & $\begin{array}{l}0 \\
0 \\
\infty \\
\infty \\
0\end{array}$ & $\begin{array}{l}\hat{f} \\
\infty \\
0 \\
0\end{array}$ & $\mid \begin{array}{l}0 \\
0 \\
0 \\
0 \\
0\end{array}$ & $\begin{array}{l}\infty \\
\infty \\
\infty \\
\infty \\
0\end{array}$ & $\begin{array}{l}\mathscr{O} \\
\infty \\
\infty \\
\infty \\
O\end{array}$ & $\begin{array}{l}\text { fo } \\
\infty \\
0 \\
0\end{array}$ & $\mid \begin{array}{l}0 \\
2 \\
m \\
m\end{array}$ & $\begin{array}{l}8 \\
8 \\
0 \\
0 \\
0\end{array}$ & 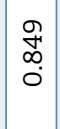 & $\begin{array}{c}\mathcal{M} \\
\infty \\
\infty \\
0\end{array}$ & 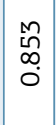 & 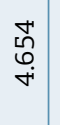 & $\begin{array}{l}0 \\
0 \\
0 \\
0\end{array}$ & $\begin{array}{l}\hat{S} \\
\stackrel{\sigma}{\rightarrow}\end{array}$ & $\begin{array}{c}\sigma \\
0 \\
\infty \\
0\end{array}$ & $\begin{array}{c}\vec{\Delta} \\
\infty \\
0 \\
0\end{array}$ & & $\begin{array}{l}\stackrel{+}{\infty} \\
\stackrel{\infty}{0} \\
0\end{array}$ & 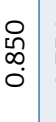 & 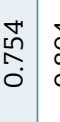 & \begin{tabular}{c|c} 
& 0 \\
$\infty$ & $a$ \\
0 & $\alpha$ \\
0 & $c$ \\
$c$
\end{tabular} & 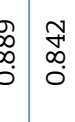 & 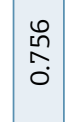 & $\begin{array}{c}\vec{N} \\
\infty \\
0 \\
0\end{array}$ & 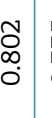 & 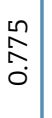 \\
\hline$\sum_{\text {R }}^{2}$ & & $\stackrel{m}{\stackrel{m}{0}}$ & $\stackrel{\stackrel{m}{\sim}}{o}$ & 움 & ণั & ஸ̆ & $\stackrel{M}{N}$ & $\begin{array}{l}\dot{0} \\
\dot{0} \\
0\end{array}$ & în & $\begin{array}{l}0 \\
-1 \\
0\end{array}$ & 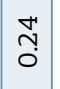 & $\overrightarrow{-1}$ & $\stackrel{f}{\circ}$ & $\begin{array}{r}\overrightarrow{1} \\
\stackrel{1}{0}\end{array}$ & ने & $\stackrel{\text { N}}{o}$ & $\underset{-}{-i}$ & 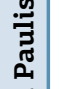 & $\begin{array}{c}\stackrel{o}{0} \\
\text { ò }\end{array}$ & mi & \begin{tabular}{l}
0 \\
\multirow{O}{O}{}
\end{tabular} & 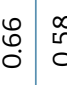 & \begin{tabular}{lll}
$\overbrace{0}^{\infty}$ & $\hat{0}$ \\
\multirow{2}{*}{}
\end{tabular} & 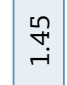 & लि & ָָ & б̆ \\
\hline 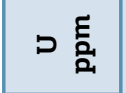 & Iึి & 点 & 8 & $\underset{\vec{I}}{\stackrel{\Im}{\sim}}$ & 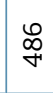 & 点 & 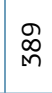 & 8 & $\stackrel{M}{Y}$ & 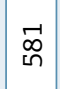 & F & $\begin{array}{l}\text { ñ } \\
\text { in }\end{array}$ & กี & $\begin{array}{l}\text { ले } \\
\text { Oे } \\
m\end{array}$ & $\begin{array}{l}\overrightarrow{1} \\
\text { in }\end{array}$ & 苂 & $\stackrel{\infty}{\tilde{N}}$ & 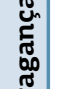 & $\begin{array}{l}\infty \\
\text { ஸ̂ } \\
\text { N }\end{array}$ & ले & $\underset{\sim}{N}$ & 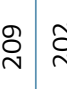 & 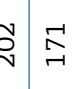 & $\underset{⿱}{\stackrel{N}{N}}$ & ت્ન & $\begin{array}{l}\tilde{m} \\
\vec{m}\end{array}$ & 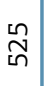 \\
\hline 톰 & ณ̃ & $\stackrel{2}{2}$ & $\underset{\sim}{\stackrel{O}{+}}$ & $\stackrel{\stackrel{n}{二}}{\exists}$ & ळ & $\stackrel{\infty}{\Sigma}$ & $\infty$ & $\stackrel{\infty}{m}$ & 占 & बू & 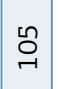 & $\infty$ & $\sqrt{2}$ & $\vec{\forall}$ & $\begin{array}{c}m \\
\infty\end{array}$ & $\hat{F}$ & $\vec{M}$ & $\begin{array}{l}m \\
0 \\
0 \\
0 \\
1\end{array}$ & $\begin{array}{l}\stackrel{0}{0} \\
\stackrel{1}{0}\end{array}$ & స̃. & & \begin{tabular}{c|c}
$\infty$ & $\vdots$ \\
\hdashline & $\vdots$ \\
0 & $\vdots$
\end{tabular} & 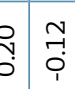 & 芯 & $\stackrel{\infty}{0}_{0}^{\infty}$ & $\begin{array}{l}0 \\
0 \\
0 \\
\end{array}$ & $\begin{array}{l}0 \\
-1 \\
0\end{array}$ \\
\hline 유뮤 & 畄 & $\begin{array}{l}0 \\
\stackrel{0}{0} \\
\text { in }\end{array}$ & $\underset{\mathfrak{z}}{\tilde{n}}$ & $\begin{array}{l}0 \\
\dot{\theta} \\
\stackrel{-}{0}\end{array}$ & $\overrightarrow{\text { जु }}$ & 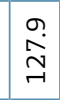 & 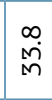 & 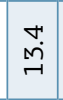 & $\stackrel{0}{\rightarrow}$ & $\stackrel{n}{\tilde{n}}$ & $\begin{array}{l}\dot{\omega} \\
\dot{\dot{m}} \\
\text { in }\end{array}$ & $\begin{array}{l}\dot{y} \\
\dot{y} \\
\dot{y}\end{array}$ & $\begin{array}{l}\dot{g} \\
\dot{g}\end{array}$ & $\begin{array}{l}\infty \\
\infty \\
\stackrel{d}{\sim} \\
\vec{\sim}\end{array}$ & $\begin{array}{l}0 \\
\dot{\theta} \\
\dot{b}\end{array}$ & $\mid \begin{array}{l}0 \\
\infty \\
\rightarrow \\
\neg\end{array}$ & $\stackrel{\curvearrowright}{\stackrel{\sim}{\sim}}$ & 空 & $\begin{array}{l}\stackrel{\circ}{i} \\
\dot{N}\end{array}$ & $\stackrel{n}{\sim}$ & 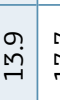 & \begin{tabular}{c|c}
$\stackrel{c}{a}$ & $\infty$ \\
\end{tabular} & 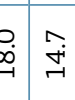 & $\hat{i}$ & $\begin{array}{l}\mathscr{\sigma} \\
\dot{\infty}\end{array}$ & $\begin{array}{l}m \\
\stackrel{M}{M} \\
\stackrel{\rho}{N}\end{array}$ & 品 \\
\hline 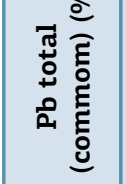 & & $\mid \begin{array}{l}0 \\
0 \\
0 \\
1\end{array}$ & $\begin{array}{l}\text { mo } \\
\dot{O}\end{array}$ & $\stackrel{-1}{0}$ & - & 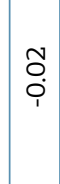 & Mn & $\begin{array}{l}0 \\
0 \\
0 \\
i\end{array}$ & 㫄 & 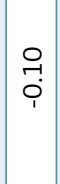 & $\begin{array}{c}-1 \\
0 \\
0\end{array}$ & $\begin{array}{l}-1 \\
0 \\
0\end{array}$ & $\begin{array}{l}\text { Mo } \\
\text { Oे }\end{array}$ & $\begin{array}{c}\tilde{N} \\
O\end{array}$ & $\overrightarrow{\vec{F}}$ & $\begin{array}{l}\text { ôn } \\
\text { ô. }\end{array}$ & $\begin{array}{l}0 \\
0 \\
0\end{array}$ & & 웅 & సี & $\underset{\rightarrow}{\vec{i}}$ & 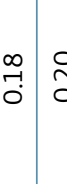 & 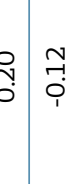 & m. & : & ن. & $\begin{array}{l}0 \\
-1 \\
0\end{array}$ \\
\hline 常 & & & $\stackrel{\vec{i}}{\mathrm{i}}$ & $N$ & & है & 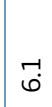 & ㄱ. & $\infty$ & $\ddot{\sigma}$ & 家 & $\stackrel{\overbrace{}}{-}$ & $\underset{\sim}{\stackrel{H}{\rightarrow}}$ & $\vec{m}$ & $\underset{\vec{H}}{\vec{H}}$ & 点 & - & & ت & 7 & $\stackrel{\sim}{\sim}$ & $\vec{m} \mid \vec{F}$ & \begin{tabular}{rl|l}
$\overrightarrow{+}$ & $\vec{H}$ \\
\end{tabular} & กู & $\overrightarrow{6}$ & $\pi$ & $\infty$ \\
\hline
\end{tabular}




\begin{tabular}{|c|c|c|c|c|c|c|c|c|c|c|c|c|c|c|c|c|c|c|c|c|c|c|c|c|c|c|c|}
\hline 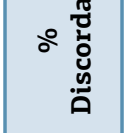 & -1 & $\sim$ & $N$ & in & $v$ & 7 & $r$ & $m$ & $\sim$ & $\vec{r}$ & $m$ & & in & $\sigma$ & $m$ & $r$ & $\varphi$ & $N$ & $m$ & $\nabla$ & $m$ & $\stackrel{M}{\rightarrow}$ & $\rightarrow$ & $\infty$ & it & $m$ & $\varphi$ \\
\hline 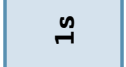 & $\tilde{m}$ & $\stackrel{H}{m}$ & 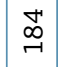 & 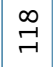 & $\vec{N}$ & $\widetilde{\sigma}$ & $\stackrel{\stackrel{M}{\sim}}{*}$ & F & $\nexists$ & $\stackrel{\leftrightarrow}{\rightarrow}$ & $\stackrel{\circ}{m}$ & & $\stackrel{\sim}{\sim}$ & $\hat{\sim}$ & i & i̊ & q & $\stackrel{M}{\sim}$ & $\stackrel{i}{\sim}$ & $\vec{n}$ & $\stackrel{N}{N}$ & $\amalg_{\infty}$ & $\stackrel{\substack{\rightarrow \\
\rightarrow}}{?}$ & $\overrightarrow{\mathbb{N}}$ & $\theta \tilde{m}$ & m & $\stackrel{\sim}{\sim}$ \\
\hline 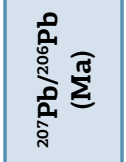 & $\begin{array}{l}0 \\
\sigma\end{array}$ & శै & $\underset{\vec{b}}{\vec{t}}$ & $\vec{\vartheta}$ & $\overrightarrow{\vec{\sigma}}$ & $\overrightarrow{6}$ & 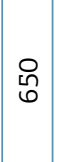 & જ̆ & : & 尺े & $\hat{\hat{o}}$ & $\begin{array}{l}x \\
\tilde{n} \\
\tilde{n}\end{array}$ & సે & $\begin{array}{l}0 \\
\delta \\
\end{array}$ & \&్ & $\begin{array}{l}\infty \\
\vec{b}\end{array}$ & $\begin{array}{l}\infty \\
0 \\
0\end{array}$ & $\begin{array}{l}0 \\
\tilde{O} \\
0\end{array}$ & $\stackrel{\mathbb{U}}{\mathbf{6}}$ & 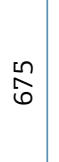 & $\stackrel{\vec{W}}{\Delta}$ & 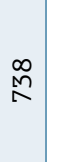 & 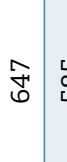 & $\begin{array}{lll}\infty & 7 \\
n \\
n\end{array}$ & $\begin{array}{l}y_{0} \\
0\end{array}$ & ర్ & $\begin{array}{l}\infty \\
\infty \\
n \\
n\end{array}$ \\
\hline$\stackrel{9}{9}$ & $\begin{array}{l}\infty \\
\end{array}$ & $\begin{array}{l}\infty \\
\stackrel{\infty}{i}\end{array}$ & $\begin{array}{l}0 \\
\dot{0}\end{array}$ & $\stackrel{\circ}{0}$ & $\begin{array}{l}0 \\
\text { ஸे }\end{array}$ & 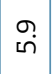 & $\begin{array}{l}6 \\
i \\
i\end{array}$ & ஸn & $\stackrel{\vec{H}}{i}$ & $\stackrel{\circ}{\circ}$ & $\begin{array}{l}\infty \\
\stackrel{\infty}{n}\end{array}$ & $\begin{array}{l}0 \\
0 \\
0 \\
0 \\
0\end{array}$ & $\stackrel{⿱ 艹}{n}$ & تn & $\stackrel{\infty}{\varphi}$ & $\begin{array}{l}\infty \\
\stackrel{\infty}{n}\end{array}$ & $\stackrel{0}{0}$ & $\stackrel{\vec{H}}{\stackrel{H}{n}}$ & $\stackrel{n}{n ்}$ & $\stackrel{m}{\varphi}$ & $\begin{array}{l}\vec{H} \\
\text { in }\end{array}$ & $\stackrel{m}{\sim}$ & $\stackrel{r}{i}$ & \begin{tabular}{l|l}
$\infty$ & \multicolumn{1}{c}{} \\
$\overrightarrow{-}$ & $\tilde{v}$
\end{tabular} & תֵ) & กู & $\stackrel{\varphi}{\stackrel{\sim}{N}}$ \\
\hline 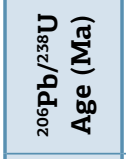 & 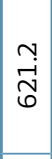 & $\begin{array}{l}\dot{0} \\
\dot{8} \\
\dot{0}\end{array}$ & $\begin{array}{l}\sigma \\
\infty \\
o \\
n n\end{array}$ & $\begin{array}{l}0 \\
\text { Oे } \\
\text { in }\end{array}$ & $\begin{array}{l}n \\
\\
0\end{array}$ & $\begin{array}{l}\overrightarrow{-} \\
\vec{\sigma} \\
\overrightarrow{6}\end{array}$ & 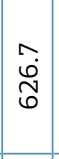 & $\underset{ت}{\stackrel{H}{-}}$ & 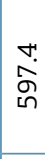 & $\begin{array}{l}\stackrel{+}{\dot{A}} \\
\stackrel{0}{0}\end{array}$ & $\begin{array}{l}0 \\
\dot{0} \\
\hat{b} \\
\end{array}$ & 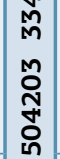 & $\begin{array}{l}0 \\
\hat{-} \\
6\end{array}$ & $\begin{array}{c}m \\
\hat{o} \\
8\end{array}$ & 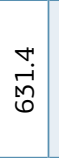 & $\begin{array}{l}0 \\
\omega \\
\vec{b} \\
\end{array}$ & $\begin{array}{l}0 \\
\dot{b} \\
\stackrel{0}{0}\end{array}$ & 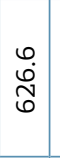 & $\begin{array}{l}\sigma \\
\vec{\nabla} \\
\vec{b}\end{array}$ & $\begin{array}{l}0 \\
- \\
0 \\
0\end{array}$ & $\begin{array}{l}\infty \\
\text { ஸीं } \\
\text { fu }\end{array}$ & $\begin{array}{l}\text { m. } \\
\vec{~} \\
\end{array}$ & 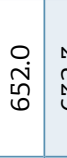 & & 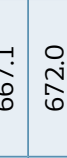 & ờ & $\begin{array}{l}\text { M } \\
\text { ஸે } \\
\text { }\end{array}$ \\
\hline$\stackrel{0}{\circ}$ & $\begin{array}{c}\tilde{N} \\
\stackrel{-}{-} \\
-1\end{array}$ & 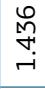 & 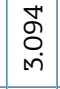 & $\begin{array}{c}m \\
\infty \\
\sim \\
\rightarrow\end{array}$ & 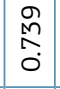 & $\begin{array}{l}0 \\
\stackrel{\leftrightarrow}{N} \\
\rightarrow \\
\sim\end{array}$ & $\begin{array}{l}0 \\
\mathscr{o} \\
-i\end{array}$ & 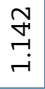 & $\stackrel{\hat{O}}{\text { i }}$ & $\begin{array}{l}\vec{\infty} \\
0 \\
0 \\
0\end{array}$ & 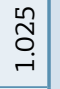 & $\stackrel{\check{\Sigma}}{\ddot{\Sigma}}$ & $\begin{array}{l}\dot{\Delta} \\
\text { Oे } \\
r\end{array}$ & $\begin{array}{c}0 \\
0 \\
0 \\
0\end{array}$ & $\begin{array}{c}\infty \\
\infty \\
\infty \\
0 \\
0\end{array}$ & $\begin{array}{c}\tilde{N} \\
\tilde{N} \\
\rightarrow\end{array}$ & 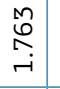 & $\begin{array}{l}\overrightarrow{\mid} \\
\text { ু } \\
\text { - }\end{array}$ & $\begin{array}{l}\infty \\
\infty \\
0 \\
0 \\
0\end{array}$ & 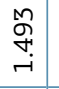 & $\begin{array}{c}\stackrel{0}{ } \\
\infty \\
\infty \\
0\end{array}$ & $\begin{array}{c}\mathcal{M} \\
\mathcal{D} \\
\rightarrow \\
-i\end{array}$ & 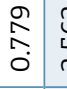 & & 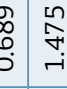 & $\underset{\substack{n \\
r}}{\tilde{r}}$ & $\begin{array}{l}\tilde{\sigma} \\
\tilde{O} \\
\text { - } \\
\text {. }\end{array}$ \\
\hline 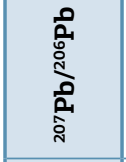 & 可 & $\begin{array}{l}\mathscr{0} \\
0 \\
0\end{array}$ & $\mid \begin{array}{l}\infty \\
0 \\
0 \\
0\end{array}$ & 怘 & $\begin{array}{l}\overrightarrow{0} \\
0 \\
0\end{array}$ & $\begin{array}{l}n \\
0 \\
0 \\
0\end{array}$ & $\begin{array}{l}\overrightarrow{0} \\
0 \\
0\end{array}$ & $\begin{array}{l}\stackrel{0}{0} \\
\stackrel{0}{\circ}\end{array}$ & : & $\begin{array}{l}\vec{b} \\
\dot{0}\end{array}$ & $\begin{array}{l}0 \\
\stackrel{0}{0} \\
0\end{array}$ & & $\begin{array}{l}\vec{b} \\
0 \\
0\end{array}$ & $\begin{array}{l}n \\
\mathscr{o} \\
0\end{array}$ & $\begin{array}{l}\overrightarrow{\breve{b}} \\
0\end{array}$ & $\begin{array}{l}0 \\
\mathscr{0} \\
0\end{array}$ & $\begin{array}{l}\overrightarrow{\ddot{b}} \\
\dot{0}\end{array}$ & $\begin{array}{l}\vec{\varnothing} \\
0 \\
0\end{array}$ & $\begin{array}{l}\stackrel{0}{0} \\
\stackrel{0}{0}\end{array}$ & $\begin{array}{l}M \\
\ddot{o} \\
0 \\
0\end{array}$ & $\begin{array}{l}\vec{b} \\
0 \\
0\end{array}$ & 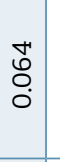 & 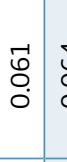 & & $\begin{array}{ll}\stackrel{0}{0} \\
\end{array}$ & $\mathscr{\delta}$ & $\begin{array}{l}\text { ô } \\
\text { ֵુ. } \\
0\end{array}$ \\
\hline 옴 & $\begin{array}{l}\text { ஸू } \\
\text { กุ } \\
0\end{array}$ & 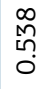 & $\begin{array}{c}0 \\
m \\
0 \\
0 \\
0\end{array}$ & 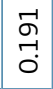 & $\begin{array}{l}\overrightarrow{0} \\
0 \\
0 \\
0\end{array}$ & $\begin{array}{c}\hat{N} \\
\tilde{N} \\
0\end{array}$ & $\begin{array}{l}\infty \\
0 \\
0 \\
0 \\
0\end{array}$ & 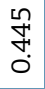 & 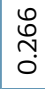 & $\begin{array}{l}\text { mo } \\
\stackrel{0}{0} \\
0\end{array}$ & $\begin{array}{l}\text { ก̂n } \\
\text { กิ }\end{array}$ & & $\begin{array}{l}0 \\
0 \\
0 \\
0\end{array}$ & $\begin{array}{l}0 \\
0 \\
0 \\
0\end{array}$ & 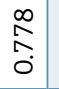 & $\begin{array}{c}\hat{N} \\
\hat{m} \\
0\end{array}$ & $\begin{array}{l}\text { 尺̃ } \\
0 \\
0 \\
0\end{array}$ & $\begin{array}{l}\mathcal{J} \\
\stackrel{S}{0} \\
0\end{array}$ & $\begin{array}{l}\tilde{n} \\
\hat{O} \\
0 \\
0\end{array}$ & $\begin{array}{c}\hat{N} \\
\text { ஸ̂. } \\
0\end{array}$ & $\begin{array}{l}\hat{n} \\
0 \\
0 \\
0\end{array}$ & 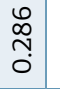 & 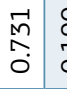 & & $\begin{array}{lll}0 & 1 \\
0 \\
0 \\
0\end{array}$ & $\begin{array}{l}\infty \\
\text { n̂. } \\
0\end{array}$ & $\begin{array}{l}\text { त゙ } \\
\text { o. } \\
0\end{array}$ \\
\hline$\stackrel{0}{\sim}$ & $\begin{array}{c}0 \\
\text { o } \\
o \\
0\end{array}$ & 今ે & $\begin{array}{c}\vec{I} \\
\vec{I} \\
\vec{i}\end{array}$ & $\begin{array}{l}\hat{0} \\
0 \\
-i\end{array}$ & $\begin{array}{l}0 \\
0 \\
\infty \\
0\end{array}$ & $\begin{array}{l}\text { g. } \\
\text { o. } \\
0\end{array}$ & 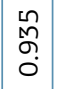 & 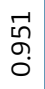 & $\begin{array}{l}0 \\
\text { ơ } \\
\circ\end{array}$ & $\begin{array}{l}\overrightarrow{0} \\
\infty \\
0\end{array}$ & $\begin{array}{l}\vec{n} \\
0 \\
0\end{array}$ & & $\begin{array}{l}n \\
\sigma \\
\sigma \\
0\end{array}$ & $\begin{array}{c}m \\
\infty \\
\infty \\
0\end{array}$ & $\begin{array}{l}\stackrel{M}{M} \\
\stackrel{H}{-}\end{array}$ & $\begin{array}{l}\Delta \\
\sigma \\
\circ \\
0\end{array}$ & $\begin{array}{c}\stackrel{M}{0} \\
\hat{O} \\
-i\end{array}$ & $\begin{array}{l}8 \\
\circ \\
\circ\end{array}$ & $\begin{array}{l}\hat{o} \\
\infty \\
0\end{array}$ & $\begin{array}{l}0 \\
-1 \\
-i\end{array}$ & $\begin{array}{l}0 \\
\infty \\
\infty \\
0 \\
0\end{array}$ & $\begin{array}{l}\infty \\
\stackrel{\infty}{-} \\
-7\end{array}$ & 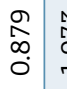 & & 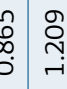 & 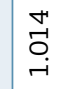 & $\begin{array}{l}\hat{T} \\
\stackrel{T}{+} \\
\end{array}$ \\
\hline 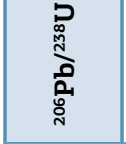 & 㕝 & $\begin{array}{l}\infty \\
0 \\
0 \\
0\end{array}$ & $\begin{array}{l}\hat{\sigma} \\
0 \\
0\end{array}$ & $\begin{array}{l}0 \\
0 \\
0 \\
0\end{array}$ & $\begin{array}{l}\infty \\
0 \\
0 \\
0\end{array}$ & $\begin{array}{l}-\overrightarrow{0} \\
\overrightarrow{1} \\
0\end{array}$ & 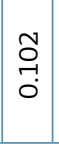 & $\begin{array}{l}\text { gे } \\
\text { Oें } \\
0\end{array}$ & ồ & $\begin{array}{l}0 \\
\text { Oे } \\
0\end{array}$ & $\begin{array}{l}\hat{0} \\
\stackrel{0}{0}\end{array}$ & & 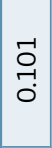 & $\begin{array}{l}0 \\
o \\
0 \\
0\end{array}$ & $\begin{array}{l}\text { Mo } \\
\stackrel{0}{0} \\
0\end{array}$ & $\begin{array}{c}8 \\
0 \\
0\end{array}$ & 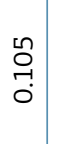 & $\begin{array}{l}\text { Oे } \\
\stackrel{-}{0}\end{array}$ & $\begin{array}{l}\text { n̊ } \\
\stackrel{0}{0} \\
0\end{array}$ & $\begin{array}{l}\mathscr{0} \\
0 \\
0 \\
0\end{array}$ & 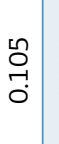 & 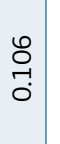 & 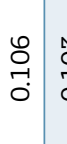 & & 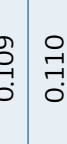 & $\begin{array}{l}\vec{~} \\
\text { Oे } \\
0\end{array}$ & $\begin{array}{l}\widetilde{\sigma} \\
\text { O- } \\
0\end{array}$ \\
\hline$\stackrel{\circ}{\circ}$ & $\begin{array}{l}0 \\
\stackrel{0}{0} \\
\end{array}$ & $\begin{array}{l}\stackrel{8}{\infty} \\
\infty \\
\rightarrow \\
\text { i }\end{array}$ & $\mid \begin{array}{l}\vec{b} \\
0 \\
\infty \\
\infty\end{array}$ & 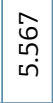 & 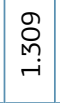 & $\begin{array}{l}\vec{n} \\
\hat{n} \\
\text { ñ. }\end{array}$ & $\begin{array}{c}\overrightarrow{\mathcal{F}} \\
\vec{i}\end{array}$ & $\stackrel{\substack{n \\
i}}{i}$ & $\begin{array}{c}0 \\
\stackrel{0}{0} \\
\text { min }\end{array}$ & $\underset{\underset{\sim}{\stackrel{M}{\sim}}}{-}$ & $\begin{array}{c}\overrightarrow{1} \\
\stackrel{6}{-} \\
\end{array}$ & & $\begin{array}{l}\hat{o} \\
\stackrel{m}{r}\end{array}$ & $\begin{array}{c}\vec{n} \\
\hat{n} \\
\rightarrow\end{array}$ & 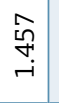 & $\begin{array}{l}\hat{\infty} \\
\stackrel{i}{i}\end{array}$ & $\stackrel{\substack{\infty \\
\stackrel{n}{N}}}{ }$ & 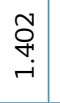 & $\underset{\vec{i}}{\vec{\Im}}$ & 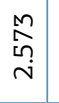 & 兽 & $\begin{array}{l}\underset{I}{\mathcal{F}} \\
\underset{+}{ }\end{array}$ & 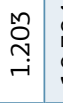 & 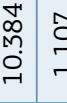 & 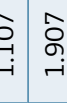 & $\begin{array}{l}\hat{\infty} \\
\stackrel{\infty}{-}\end{array}$ & 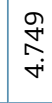 \\
\hline 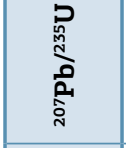 & $\begin{array}{l}\text { ڤ్ } \\
\infty \\
0 \\
0\end{array}$ & $\begin{array}{l}\vec{F} \\
\infty \\
0 \\
0\end{array}$ & $\mid \begin{array}{l}0 \\
0 \\
0 \\
0\end{array}$ & $\begin{array}{l}8 \\
\infty \\
\infty \\
0\end{array}$ & $\begin{array}{c}0 \\
\infty \\
\infty \\
0\end{array}$ & $\begin{array}{c}\hat{m} \\
\infty \\
0 \\
0\end{array}$ & $\begin{array}{l}n \\
\tilde{o} \\
\infty \\
0\end{array}$ & $\begin{array}{c}\infty \\
\infty \\
\infty \\
0 \\
0\end{array}$ & مْ & $\begin{array}{l}0 \\
\infty \\
0 \\
0\end{array}$ & 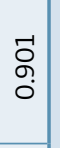 & & $\begin{array}{c}0 \\
\text { 足 } \\
\infty \\
0 \\
0\end{array}$ & $\begin{array}{c}0 \\
1 \\
\infty \\
0\end{array}$ & $\begin{array}{l}0 \\
0 \\
\infty \\
0 \\
0\end{array}$ & $\begin{array}{c}m \\
\infty \\
\infty \\
0\end{array}$ & $\begin{array}{c}\stackrel{\nabla}{ } \\
\infty \\
0 \\
0\end{array}$ & $\begin{array}{l}\hat{n} \\
\infty \\
0 \\
0\end{array}$ & $\begin{array}{c}\stackrel{+}{ } \\
\infty \\
0 \\
0\end{array}$ & 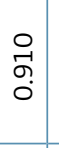 & 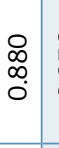 & $\begin{array}{c}0 \\
\stackrel{0}{ } \\
o \\
o\end{array}$ & $\begin{array}{l}\mathscr{g} \\
\infty \\
0 \\
0\end{array}$ & & \begin{tabular}{c}
0 \\
\multirow{2}{*}{} \\
\end{tabular} & $\begin{array}{c}\sqrt{1} \\
\text { o } \\
0\end{array}$ & $\begin{array}{c}\hat{N} \\
\infty \\
\infty \\
0\end{array}$ \\
\hline $\begin{array}{l}\text { R } \\
\text { T }\end{array}$ & $\begin{array}{l}\text { Da } \\
\infty \\
0\end{array}$ & $\begin{array}{l}\text { ำ } \\
\text { o }\end{array}$ & 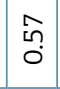 & กี่ & ণัণ & $\begin{array}{c}\text { m. } \\
o \\
o\end{array}$ & $\begin{array}{l}0 \\
0 \\
0\end{array}$ & 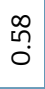 & 苞 & $\begin{array}{c}0 \\
m \\
0\end{array}$ & $\begin{array}{l}\stackrel{M}{\hat{O}} \\
\hat{0}\end{array}$ & $\begin{array}{l}\bar{U} \\
\text { ठ } \\
\tilde{J}\end{array}$ & ๕̊ & $\begin{array}{c}\tilde{m} \\
\tilde{O} \\
0\end{array}$ & 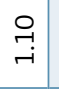 & 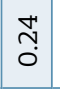 & $\begin{array}{l}\mathscr{8} \\
\dot{0}\end{array}$ & $\begin{array}{c}\mathcal{M} \\
\hat{O}\end{array}$ & 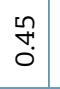 & 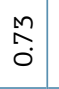 & 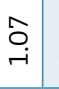 & $\begin{array}{l}\stackrel{\overbrace{}}{\rightarrow} \\
\stackrel{-}{~}\end{array}$ & 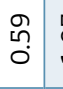 & \begin{tabular}{l|l}
$\hat{\infty}$ & $\stackrel{\alpha}{c}$ \\
$c$
\end{tabular} & 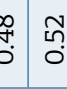 & $\underset{\sim}{\tilde{r}}$ & f̆ \\
\hline D & $\stackrel{\vec{N}}{\sim}$ & $\begin{array}{l}\overrightarrow{0} \\
\stackrel{-}{*}\end{array}$ & $\vec{m}$ & $\stackrel{\infty}{\stackrel{\infty}{\sim}}$ & 斊 & 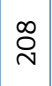 & 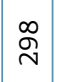 & $\stackrel{0}{\stackrel{0}{N}}$ & $\underset{\sim}{\sim}$ & $\tilde{\mathscr{O}}$ & $\overrightarrow{\widehat{N}}$ & 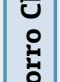 & $\stackrel{\infty}{\sim}$ & $\stackrel{\mathscr{f}}{f}$ & $\underset{f}{\mathscr{g}}$ & $\stackrel{m}{\sim}$ & $\underset{\mathbb{Z}}{\mathbb{Z}}$ & 品 & 命 & $\stackrel{0}{0}$ & $\begin{array}{l}\text { Oु } \\
\text { bे }\end{array}$ & n̊ & $\begin{array}{l}m \\
n \\
n\end{array}$ & $\begin{array}{l}\infty \\
\sigma \\
\end{array}$ & 党 & $\stackrel{\mathscr{\sigma}}{\circ}$ & 尽 \\
\hline 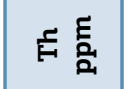 & $\begin{array}{l}\infty \\
0 \\
0\end{array}$ & $\begin{array}{l}\infty \\
\stackrel{-}{0}\end{array}$ & 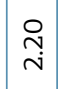 & $\stackrel{\widehat{N}}{\rightarrow}$ & $\begin{array}{l}8 \\
0 \\
0\end{array}$ & $\begin{array}{l}0 \\
0 \\
0\end{array}$ & O & o̊. & $\stackrel{n}{\stackrel{n}{o}}$ & नें & $\begin{array}{l}\overrightarrow{7} \\
\stackrel{1}{1} \\
\end{array}$ & $\begin{array}{l}\text { ò } \\
\text { जे }\end{array}$ & $\underset{\sim}{\stackrel{\sim}{\sim}}$ & $\begin{array}{l}\infty \\
\varrho \\
\ddots\end{array}$ & $\overrightarrow{n ̃ n}$ & $\mathscr{f}$ & $\infty$ & $\begin{array}{l}\text { n̂ } \\
\text { m }\end{array}$ & $\stackrel{M}{\stackrel{M}{\sim}}$ & $\underset{ન}{\stackrel{\vartheta}{二}}$ & $\begin{array}{l}\stackrel{0}{\forall} \\
\stackrel{\gamma}{\prime}\end{array}$ & ને & $\stackrel{M}{\stackrel{N}{N}}$ & $\infty \stackrel{0}{\infty}$ & & $\stackrel{\infty}{\stackrel{\infty}{N}}$ & $\underset{\sim}{\mathscr{I}}$ \\
\hline م ت己 & $\begin{array}{l}0 \\
\stackrel{\sim}{N}\end{array}$ & $\begin{array}{l}\infty \\
\stackrel{\infty}{\rightarrow} \\
\text { - }\end{array}$ & $\underset{\sim}{\stackrel{N}{G}}$ & $\underset{⿱}{\stackrel{O}{+}}$ & $\begin{array}{l}\infty \\
0 \\
i \\
i n\end{array}$ & $\begin{array}{c}-1 \\
\infty \\
-1\end{array}$ & 㫄 & $\begin{array}{l}\stackrel{\leftrightarrow}{\sim} \\
\stackrel{\text { N }}{ }\end{array}$ & 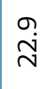 & $\underset{\infty}{\stackrel{\sigma}{+}}$ & 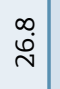 & 窗 & $\begin{array}{c}M \\
\infty \\
\stackrel{\infty}{N}\end{array}$ & $\begin{array}{l}0 \\
\infty \\
m\end{array}$ & $\underset{H}{+}$ & $\begin{array}{c}n \\
\\
\end{array}$ & $\underset{ت}{\vec{G}}$ & ì & ç & $\begin{array}{l}m \\
\stackrel{n}{n}\end{array}$ & $\begin{array}{l}\stackrel{0}{\vec{r}} \\
\dot{\vec{r}}\end{array}$ & $\widehat{\infty}$ & $\begin{array}{l}\mathscr{g} \\
\dot{\psi}\end{array}$ & 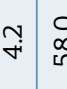 & 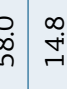 & ڤે & o \\
\hline 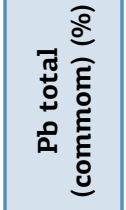 & $\begin{array}{l}\infty \\
0 \\
0\end{array}$ & $\stackrel{\infty}{\stackrel{\infty}{\sim}}$ & ্ָণ & 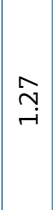 & $\begin{array}{l}8 \\
0 \\
0\end{array}$ & $\begin{array}{l}0 \\
0 \\
0\end{array}$ & ৪ & o̊ & مُ & ने & $\begin{array}{l}\stackrel{ \pm}{7} \\
\stackrel{1}{i}\end{array}$ & & $\vec{s}$ & 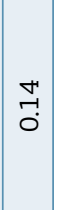 & ö & $\begin{array}{r}\vec{H} \\
\stackrel{1}{0}\end{array}$ & \begin{tabular}{c}
$\stackrel{n}{n}$ \\
\hdashline \\
0
\end{tabular} & ơ & $\begin{array}{l}\text { Mo } \\
0 \\
\\
1\end{array}$ & ને & ồ & $\begin{array}{l}\text { ñ } \\
\text { O. }\end{array}$ & ö & 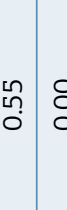 & : & ֻُ & $\begin{array}{l}-1 \\
0 \\
0\end{array}$ \\
\hline 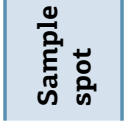 & & & & vis & $\left|\begin{array}{c}\mathfrak{j} \\
\vec{H}\end{array}\right|$ & & $\underset{\stackrel{+}{+}}{\vec{f}}$ & $\vec{H}$ & | & & $\overrightarrow{\overrightarrow{7}}$ & & & $\vec{i}$ & $\vec{m}$ & $\stackrel{\vec{F}}{\vec{F}}$ & $\vec{n}$ & $\overrightarrow{6}$ & $\stackrel{-!}{r}$ & $\vec{\infty}_{\infty}^{-1}$ & б̆ & 㖻 & $\vec{I}$ & $\begin{array}{c}\overline{7} \\
\vec{F}\end{array}$ & $\begin{array}{c}\Delta \\
\mathbb{H}\end{array}$ & $\vec{m}$ & \\
\hline
\end{tabular}


$\pm 32 \mathrm{Ma}$. The remaining points spread over the Concordia for a relatively wide age range. The weighted average ${ }^{206} \mathrm{~Pb} /{ }^{238} \mathrm{U}$ age of all thirteen point results in a large error $(615 \pm 11 \mathrm{Ma})$ and high MSWD (4.2); together, these points do not yield a concordia. Excluding the two older results, which do not seem to be part of a continuous with the others, yields a weighted average ${ }^{206} \mathrm{~Pb} /{ }^{238} \mathrm{U}$ age of $609.9 \pm 8.4 \mathrm{Ma}$ (at the $95 \%$ confidence level) with MSWD = 2.1 and very low probability of fit (0.025) (Fig. 6). These spots yield a concordia age of $608.3 \pm 6.6 \mathrm{Ma}$ with MSWD $=2.2$ and probability of fit $=0.14$. This is considered as the best age estimate of magmatic crystallization of sample ATI-13. A regression considering only the nine youngest results yields a slightly younger age $(606.2 \pm 5.5 \mathrm{Ma})$ with much lower MSWD (0.19) and higher probability of fit, but we see no criteria to exclude spots 1.1 and 3.1. The ages obtained for the Bragança Paulista granite using the two different approaches are thus identical within error.

\section{Porphyritic biotite-syenogranite (Salmão-type)}

\section{Sample petrography and geochemistry}

The porphyritic biotite-syenogranite corresponds to the Salmão association (Campos Neto et al. 1984a) or the Socorro II magmatism of Artur et al. (1993). These granites form plutons in different portions of the batholith, with dimensions varying from 2 to $25 \mathrm{~km}^{2}$.

Sample BRP-03 was collected in the southern portion of the batholith (Fig. 2) and corresponds to an inequigranular porphyritic pink-colored biotite syenogranite with a color index around 6 and alkali feldspar megacrysts measuring, on average, $4 \times 1.5 \mathrm{~cm}$ (Fig. 3B). Accessory minerals include apatite, zircon, opaque minerals and monazite.

The geochemical data show high contents of $\mathrm{SiO}_{2}$ (73.6 wt\%), $\mathrm{K}_{2} \mathrm{O}$ (5.2 wt\%), Th (35 ppm) and $\mathrm{U}$ (4 ppm) (Fig. 7); mg\# is -30 . In comparison to the other samples dated, it has the lowest contents of $\mathrm{Fe}_{2} \mathrm{O}_{3}(1.3 \mathrm{wt} \%), \mathrm{MnO}$ $(<0.02 \mathrm{wt} \%)$ and $\mathrm{MgO}(0.3 \mathrm{wt} \%)$. The REE pattern is very fractioned, with $(\mathrm{La} / \mathrm{Yb})_{\mathrm{N}}=166$ with a negative anomaly of $\mathrm{Eu}\left(\mathrm{Eu} / \mathrm{Eu}^{*}=0.7\right)$ (Fig. 5).

\section{Zircon morphology and $\mathrm{U}-\mathrm{Pb}$ dating}

Zircons from sample BRP-03 can be divided into two morphologically distinct populations. One has elongated prismatic shape with up to $300 \mu \mathrm{m}$ length, and 3:1 aspect ratio. The cores are heterogeneous, without zoning or exhibiting inconspicuous and irregular zoning, and are typically darker than rims. The other population has more rounded shapes and lengths up to $200 \mu \mathrm{m}$, with some cores, in CL images (Suppl. data), with a bright appearance.
Three cores yield inherited ages, but spot 14.1 is strongly discordant and will not be further considered. Spot 12.1 is $98 \%$ concordant, and yields a ${ }^{207} \mathrm{~Pb} /{ }^{206} \mathrm{~Pb}$ age of $1,777 \pm$ $42 \mathrm{Ma}$; spot 7.1 is $12 \%$ discordant, and its ${ }^{207} \mathrm{~Pb} /{ }^{206} \mathrm{~Pb}$ age is similar within error $(1,675 \pm 98 \mathrm{Ma})$.

Four of the other 13 points are $>+10 \%$ discordant, and as such show ${ }^{206} \mathrm{~Pb} /{ }^{238} \mathrm{U}$ that are too young. The remaining nine spots yield a weighted average ${ }^{206} \mathrm{~Pb} /{ }^{238} \mathrm{U}$ age (Fig. 6) of $626.6 \pm 6.4 \mathrm{Ma}$ with high MSWD = 2.4. Spot 15.1 has the highest age and is not part of a coherent age group with the other eight spots. Excluding it, the weighted average is 624.7 $\pm 3.6 \mathrm{Ma}(2 \sigma)$ with a lower MSWD (1.04) and a probability of fit of 0.46 . These eight samples yield a Concordia age (Fig. 8) of 624.4 $\pm 3.6 \mathrm{Ma}$ (MSWD = 0.24), which is considered the magmatic crystallization age of sample BRP-03.

\section{Charnockites}

\section{Sample petrography and geochemistry}

Charnockites were described as a marginal facies with transitional contacts to porphytic granites associated to the Socorro I magmatism nearby the city of Socorro (Artur 2003, Wernick et al. 1984a), occurring as elongated bodies with a maximum extension of $20 \mathrm{~km}$. Sample BRP-12 was collected in this region, and is a foliated, porphyritic charnockite with dark green colour, and alkali feldspar as $2 \times 1 \mathrm{~cm}$ megacrysts, and as a matrix mineral with plagioclase, quartz, orthopyroxene, clinopyroxene, biotite and hornblende.

The Atibaia Charnockite is a small NE-elongated body $(3 \times 0.5 \mathrm{~km})$ in the southern portion of the Socorro Batholith (Fig. 2). Sample ATB-08 is medium- to coarse-grained, foliated, with greenish-brown color and monzogranitic composition Mafic minerals are clinopyroxene, orthopyroxene, amphibole and traces of biotite; accessory minerals are zircon, apatite and opaque minerals.

Our geochemical data (Fig. 4) shows important differences between the two charnockite occurrences. The Socorro charnockite sample is relatively primitive, and its geochemical signature is very similar to the Bragança Paulista-type granites, with $61.6 \mathrm{wt} \% \mathrm{SiO}_{2}$, 4.6 wt $\% \mathrm{CaO}, 2.2 \mathrm{wt} \% \mathrm{MgO}$ and $\mathrm{mg \#}$ - 43, combining relatively high contents of $\mathrm{K}_{2} \mathrm{O}(3.7 \%)$, $\mathrm{Ba}(1,300$ ppm), Sr (800 ppm) and a fractionated REE pattern $\left((\mathrm{La} / \mathrm{Yb})_{\mathrm{N}}=82\right)$ with poorly developed negative Eu anomaly $\left(\mathrm{Eu} / \mathrm{Eu}^{*}=0.84\right)$ (Fig. 5). The Atibaia charnockite has higher contents of $\mathrm{SiO}_{2}(70 \mathrm{wt} \%), \mathrm{K}_{2} \mathrm{O}$ (5.8 wt\%), and $\mathrm{Zr}(402 \mathrm{ppm})$ and much lower $\mathrm{Sr}(170 \mathrm{ppm})$; the REE patterns are moderately fractionated $\left((\mathrm{La} / \mathrm{Yb})_{\mathrm{N}}=\right.$ 32.3) with a more pronounced negative Eu anomaly $\left(\mathrm{Eu} / \mathrm{Eu}^{*}=0.62\right)($ Fig. 5). This chemical signature is 
similar to neighboring garnet-bearing biotite granites (Fig. 5), and different from both Bragança Paulista and Salmão-type granites.

Bragança Paulista (BRP - 08)

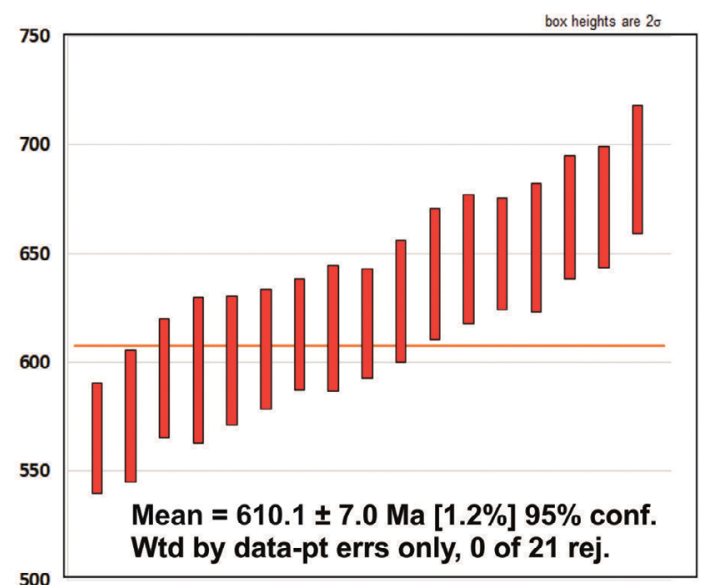

Salmão (BRP - 03)

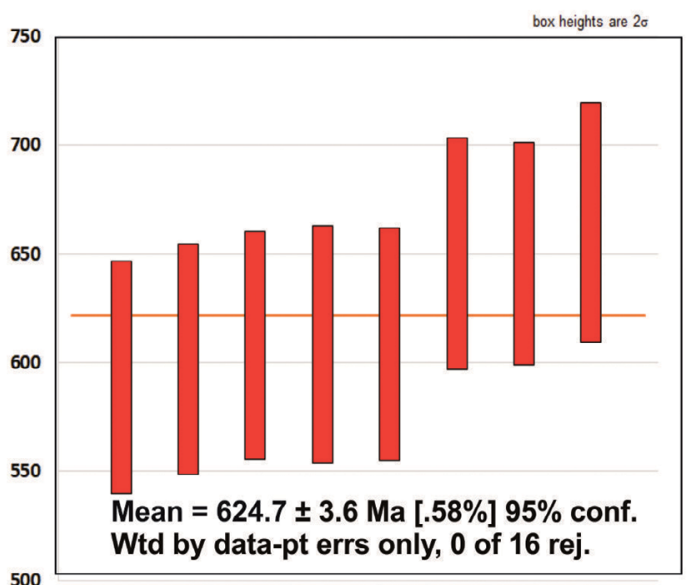

Zircon morphology and $\mathrm{U}-\mathrm{Pb}$ dating

Zircon crystals from sample BRP-12 have different sizes and shapes, and can be divided into two populations, based

Atibaia Charnockite (ATB - 08)

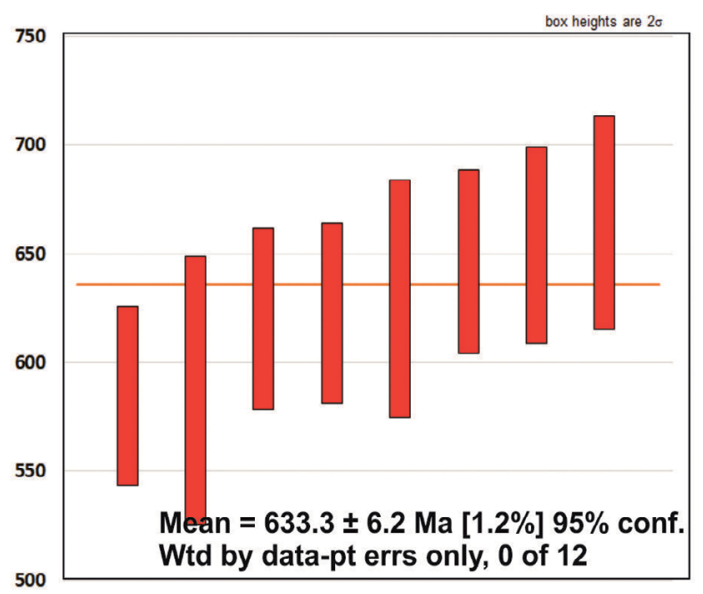

Figure 6. Weighted average of ${ }^{206} \mathrm{~Pb} / 238 \mathrm{U}$ ages of dated zircon spots. 
on morphological and textured grain differences. The first is elongated, prismatic, with lengths of $200-350 \mu \mathrm{m}$; in CL images (Suppl. data), they show rims with oscillatory zoning and cores with dark gray to white shading. Crystals from the second population are $150-200 \mu \mathrm{m}$ in length, with cores typically brighter (lower $\mathrm{U}$ ) than the rims, some having a shiny appearance. In this case, the rims have little obvious zoning.

Sixteen points were analyzed and are $\leq 6 \%$ discordant, with four exceptions that were excluded from age calculations; spot 14.1 has a high analytical error and was also excluded (Tab. 3). The remaining 11 results spread along the Concordia for a wide time interval $\left({ }^{206} \mathrm{~Pb} /{ }^{238} \mathrm{U}\right.$ ages $=615-$ $667 \mathrm{Ma})$. As a result, the weighted average has a large error and MSWD (640 $\pm 11 \mathrm{Ma}$; 95\% conf.; MSWD = 7.8), and no Concordia age is obtained from this set of data. Exclusion of the oldest and the two youngest ${ }^{206} \mathrm{~Pb} /{ }^{238} \mathrm{U}$ ages defines a coherent group of 8 samples, which yields a weighted average (Fig. 6) ${ }^{206} \mathrm{~Pb} /{ }^{238} \mathrm{U}$ age of $642.0 \pm 7.7 \mathrm{Ma}$ (95\% conf.), $\mathrm{MSWD}=2.5$ and probability of fit $=0.016$. This group defines a Concordia age (Fig. 8) of $641.6 \pm 4.1 \mathrm{Ma}(2 \sigma)$, MSWD = 0.5 and probability of fit $=0.48$, which is considered the best estimate of the age of magmatic crystallization of the sample.

Zircon crystals from sample ATB-08 are elongated, with rare rounded shapes, and lengths up to $420 \mu \mathrm{m}$. The cores present oscillatory zoning or are homogeneous, rarely with bright aspect and rounded formats in CL images (Suppl. data). In addition, some cores have inclusions and shades of dark gray or black. The rims may show some zoning.

Two out the 12 spots analyzed are $>5 \%$ discordant $\left(-7\right.$ and $+9 \%$; Table 3). A weighted average ${ }^{206} \mathrm{~Pb} /{ }^{238} \mathrm{U}$ age obtained with all 12 spots yields $634.8 \pm 5.6 \mathrm{Ma}$ with MSWD $=1.5$ and probability of fit $=0.11$. A Concordia age can be obtained with all these results and yields 631.5 $\pm 6.7 \mathrm{Ma}(95 \%$ confidence $)$, with a much higher MSWD =

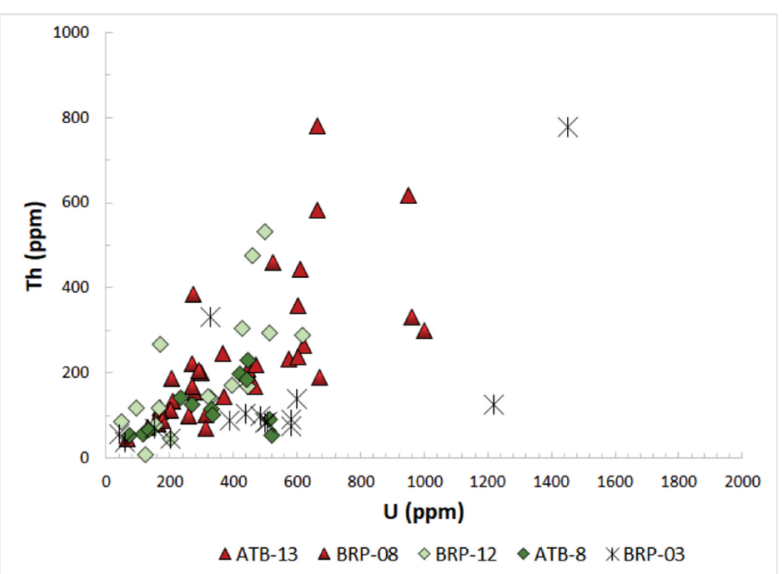

Figure 7. Geochemical variation diagram Th vs. U. of dated samples.
7.6 and very low probability of fit (0.006). Excluding the two results that are $>5 \%$ discordant yields a weighted average ${ }^{206} \mathrm{~Pb} /{ }^{238} \mathrm{U}$ age of $633.3 \pm 6.2 \mathrm{Ma}(2 \sigma)$ with MSWD = 1.6 and probability of fit $=0.096$. A Concordia age (Fig. 8) obtained from these 10 results yields an age of $630.3 \pm$ 5.7 Ma, with high MSWD (5.1) and low probability of fit (0.024). All these ages are coincident within error; the weighted average (Fig. 6) ${ }^{206} \mathrm{~Pb} /{ }^{238} \mathrm{U}$ age of $633.3 \pm 6.2 \mathrm{Ma}$ is admitted as the best estimate of the age of this sample.

\section{DISCUSSION}

\section{Age of granitic magmatism in Socorro Batholith}

Five samples of the most typical granite types of the Socorro Batholith were chosen for U-Pb dating by SHRIMP in this study. The samples consist of porphyritic granites related in previous works to the Bragança Paulista and Salmão suites (respectively, Socorro I and Socorro II associations of Artur et al. 2013) and two charnockites that form small occurrences described as transitional to granites from the batholith.

The ages are interpreted as of magmatic crystallization and show a wide time interval, from early charnockites at -642 Ma to high-K calc-alkaline metaluminous (biotite-hornblende) granites (Bragança Paulista-type) at $-610 \mathrm{Ma}$, i.e., a minimum 30 M.y. interval for the build up of the batholith.

The five ages obtained here seem part of a continuum and suggest a chronological sequence from the Socorro Charnockite $(641.6 \pm 4.1 \mathrm{Ma})$ to the Atibaia Charnockite $(633.3 \pm 6.2 \mathrm{Ma})$, Salmão-type granite $(624.4 \pm 3.6 \mathrm{Ma})$, and then the Bragança Paulista-type granites (610.1 \pm 7.0 $\mathrm{Ma}$ and $608.3 \pm 6.6 \mathrm{Ma}$ ). This sequence contradicts some of the assumptions made in the literature.

The Socorro Charnockite is described as transitional with the Socorro I (Bragança Paulista-type) granites and yet has a much older age. It is possible that the granites described as transitional to the Socorro Charnockite (which remain so far undated) are indeed of the same age, and in fact their geochemical signature (Wernick et al. 1984a) is very similar to the Bragança Paulista type granites, but our data suggest that the Socorro charnockites (and eventually their opx-free equivalents) may belong to an older association.

The fact that the dated "Salmão-type" granite is older than the two Bragança Paulista type granites may appear surprising, since this type of granite is reported as younger in the literature ("Socorro II" association), based on field work (Artur et al. 1993, 1994, 1996, Wernick et al. 1997, Wernick \& Menezes 2001, Artur 2003, Campos Neto et al. 1984b). Again, older HKCA (Bragança Paulista-type) granites may exist, and this is clear from the U-Pb zircon TIMS 
Bragança Paulista (BRP - 08)

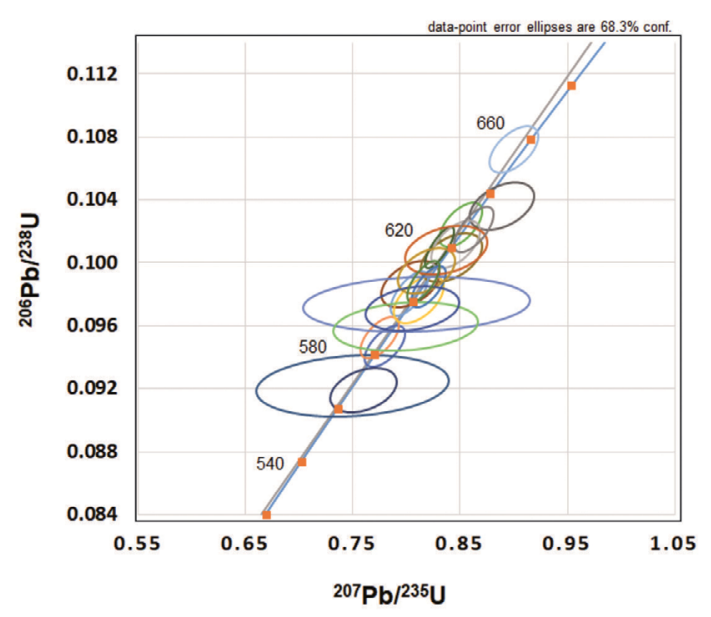

Salmão (BRP - 03)

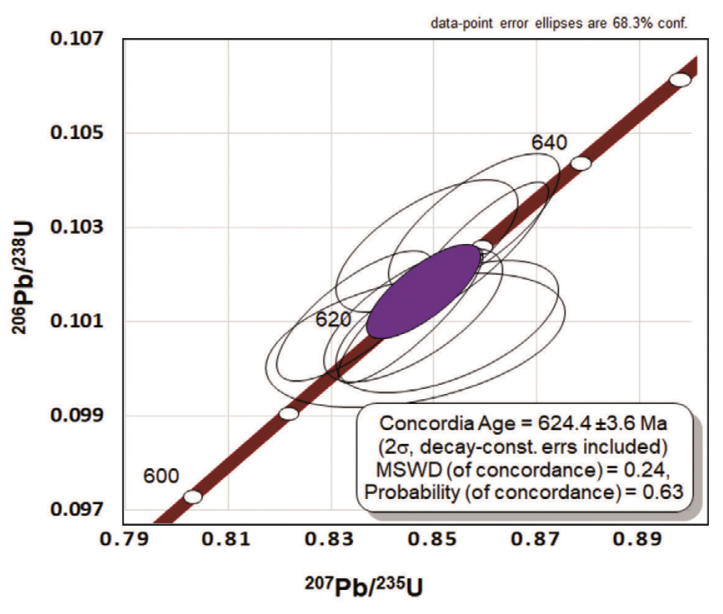

Socorro Charnockite (BRP - 12)

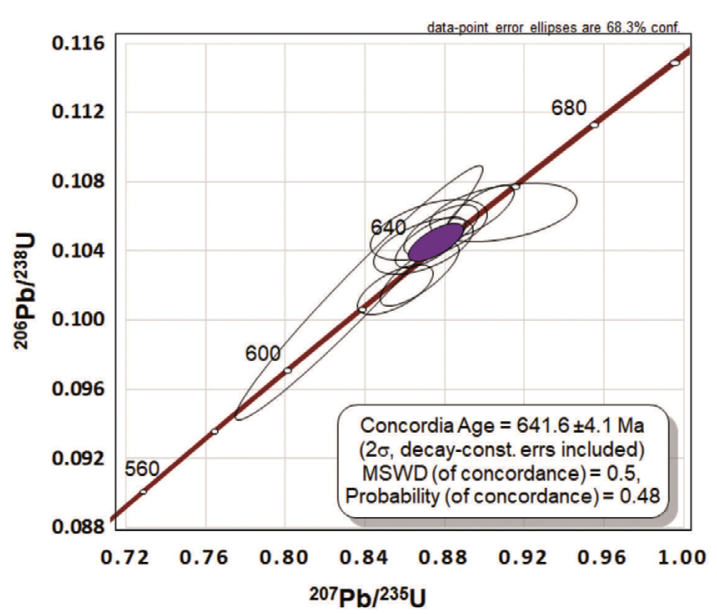

Bragança Paulista (ATB -13)

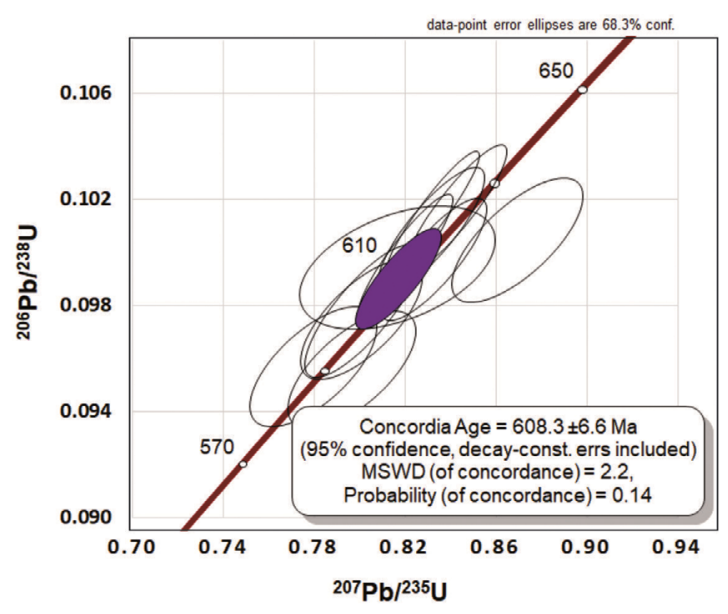

Salmão (BRP - 03 - all points)

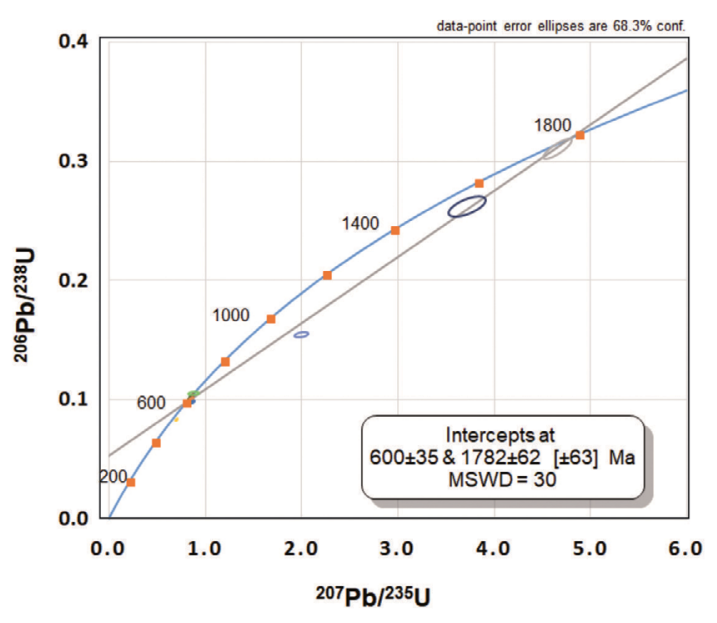

Atibaia Charnockite (ATB - 08)

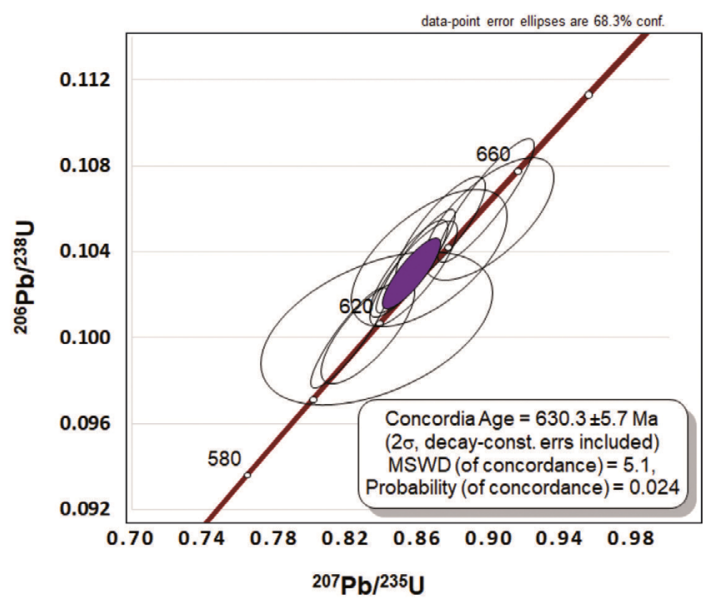

Figure 8. Concordia ages of dated samples, with an additional figure from sample BRP-03 indicating the points with inherited ages. Data for BRP-08 do not yield a Concordia age, and in this figure only the graph with age distribution are shown. 
age reported by Topfner (1996) for a sample with typical Bragança Paulista affinity $(629 \pm 3 \mathrm{Ma})$. While the scrutiny of the data for this sample in the original work does not show any suggestions that a different interpretation could be offered for its age, we observe that some other ages reported in that work were shown to be -20 M.y. older than the ages obtained for the same plutons in a recent SHRIMP and LA-ICPMS dating program (Janasi et al. 2016), and this was attributed to the presence of tiny inclusions of inherited zircon in the multigrain fractions used in the TIMS study. Our ages for the two Bragança Paulista-type granites are identical within error, and coincide with the age reported by Ebert et al. (1996) to a sample collected in the southeastern portion of the batholith $(610 \pm 10 \mathrm{Ma})$.

Except in sample BRP-03 (Salmão-type granite), inheritance is uncommon in the studied samples; in fact, no inherited zircon was identified in the two charnockite samples and a single one was found in one of the Bragança-Paulista type granites (BRP-13). This suggests that the effect reported by Janasi et al. (2016) in the São Roque granites should not be effective here. The spread of ages over the concordia for up to 50 M.y. (also recorded in similar granites in the region, see for instance Vinagre et al. 2014) may reflect other two causes: the presence of antecrysts (i.e., crystals from the same magmatic system previously crystallized and cannibalized by the magma that formed the dated pulse) or slightly younger lead-loss events (as demonstrated by several recent reports of U-Pb zircon ages dated by TIMS after chemical abrasion; e.g., Almeida et al. 2018). At the moment, we cannot detect these effects, and thus we admit that both may respond for part of the spread. TIMS dating of selected crystals after chemical abrasion would certainly be a valuable tool to further improve the geochronology of some samples.

\section{Correlations with other batholiths of HKCA affinity in SE Brazil}

The Socorro Batholith is one of the major expressions of Neoproterozoic granitic magmatism in SE Brazil and is comparable in volume to other important batholiths dominated by HKCA granites such as Agudos Grandes, Três Córregos, Cunhaporanga and Serra da Água Limpa. Figure 9 compares the ages obtained in this work with data obtained by the $\mathrm{U}-\mathrm{Pb}$ method in zircon for these batholiths.

Only recently in situ methods (SHRIMP and LA-ICPMS) have been used to date these granites, and as reported by Janasi $e t$ al. (2016), at least in the case of the granites from the São Roque Domain, the new in situ results have shown systematically younger ages, which raised the suspicion that some ages obtained by TIMS may be older than the true magmatic age (by as far as
20 M.y.) due to the presence of tiny inclusions of inherited zircon in multigrain fractions used for dating. A similar situation was observed in the geochronology of HKCA granites from the Três Corregos and Cunhaporanga granites, where part of the U-Pb TIMS ages older than 610 Ma presented in preliminary reports (Prazeres Filho et al. (2003) were revised to younger values $(610-590 \mathrm{Ma})$ after SHRIMP dating (Prazeres Filho 2006). In fact, important amounts of zircon inheritance are reported in samples of HKCA granites from these batholiths, particularly those intruding metasedimentary sequences in the Ribeira Belt (Três Córregos, Cunhaporanga and Agudos Grandes (e.g., Gimenez Filho et al. 2000, Janasi et al. 2001, Prazeres Filho et al. 2003, Leite et al. 2007), and in situ methods seem more adequate to obtain their true age of magmatic crystallization.

Figure 9 shows that the best estimates of magmatic crystallization ages available for HKCA granites from the Ribeira Belt indicate that the greatest volume of these granites was generated in the 610-590 Ma period. In the Agudos Grandes batholith, Leite et al. (2007) suggested that the typical HKCA granites forming elongated intrusions are slightly older than equivalents more contaminated with middle crust material that form plutons with subcircular shape in plant, referred to as "late-orogenic", whose ages are in the 605-600 Ma interval. In the São Roque Domain, typical HKCA granites also tend to be slightly older than occurrences with peraluminous or subalkaline character dated at $-590 \mathrm{Ma}$ (Janasi et al. 2016).

Age determinations for the HKCA magmatism in the Socorro-Guaxupé are less abundant in the literature, and the results of LA-ICPMS U-Pb dating reported for the Serra da Água Limpa Batholith by Vinagre et al. (2014) indicate an older interval for the HKCA granite magmatism (645-630 Ma; Fig. 9). This partly overlaps the range of ages determined for the migmatitic orthogneisses admitted to be subduction-related by Hackspacher et al. (2003) (660-640 Ma). Our new data indicates that important volumes of Bragança Paulistatype granites were generated at ca. $610 \mathrm{Ma}$ in the Socorro Batholith (see also the age reported by Ebert et al. 1996), i.e., contemporaneous with the peak of HKCA magmatism in the nearby (Apiaí and São Roque) domains of the Ribeira Belt.

\section{Inferences on sources and tectonic environment}

A reassessment of the petrogenesis of the southern part of the Socorro Batholith is the subject of a current project using additional elemental and isotope geochemistry data, and is not the focus of the present article. A few considerations can be made considering 
the whole-rock geochemical and geochronological data presented here.

The oldest age obtained here is that of the Socorro Charnockite, which has a HKCA geochemical signature very similar to the Bragança Paulista-type granites. These dry, high-temperature, relatively primitive $\left(-62 \mathrm{wt} \% \mathrm{SiO}_{2}\right)$ are not typical products of partial melting of the continental crust, and may have connections with mantle-derived basic magmatism, which is also suggested by the common presence of mafic enclaves.

The age obtained for the Atibaia Charnockite is ca. $10 \mathrm{Ma}$ younger, and the chemistry of this rock (and also of associated granites, Fig. 4) is indeed indicative of a different source and perhaps also of a change in tectonic setting. The relatively low $\mathrm{mg \#}-27$ combined with high $\mathrm{Zr}(-400 \mathrm{ppm})$ and low $\mathrm{Sr}(<180 \mathrm{ppm})$ is indicative of a non-calc-alkaline character, and perhaps a closer affinity to the São José do Rio Pardo mangerite-charnockite association present in the northern part of the NESG and dated at $623.3 \pm 3.1 \mathrm{Ma}$ (Janasi 2002).

The high-grade metamorphism that affected the metasupracrustal sequences of the SGN has been shown to have lasted for a period as long as 25 M.y., between
635 and $610 \mathrm{Ma}$, based on U-Pb and chemical dating of monazite and zircon from migmatites (Martins et al. 2009, Rocha et al. 2017). This coincides with the range of ages of the Socorro granites, with the possible exception of the Socorro Charnockite. The Atibaia Charnockite may be a product of partial melting of a dry, granulitic source, during this high-grade metamorphism, as suggested for the São José do Rio Pardo association. Similarly, the fractionated ( $\left.73 \mathrm{wt} \% \mathrm{SiO}_{2}\right)$ Salmãotype granite dated here is probably a product of crustal melting, as suggested by its moderately peraluminous character, trace-element signature (e.g., high $\mathrm{Th}$ and LREE contents) and abundance of inherited zircon. Indeed, it shares important compositional attributes with the regional anatectic granites of Nazaré Paulista type, and is coeval with the oldest occurrences of these granites (e.g., $623.6 \pm 1.6 \mathrm{Ma}$; U-Pb TIMS monazite age reported by Janasi (1999).

The - $610 \mathrm{Ma}$ ages determined for the Bragança Paulistatype HKCA granites (this work; Ebert et al. 1996) indicate that their emplacement was coeval with the late stages of regional high-grade metamorphism which is associated to partial melting of fertile crustal protoliths, possibly under

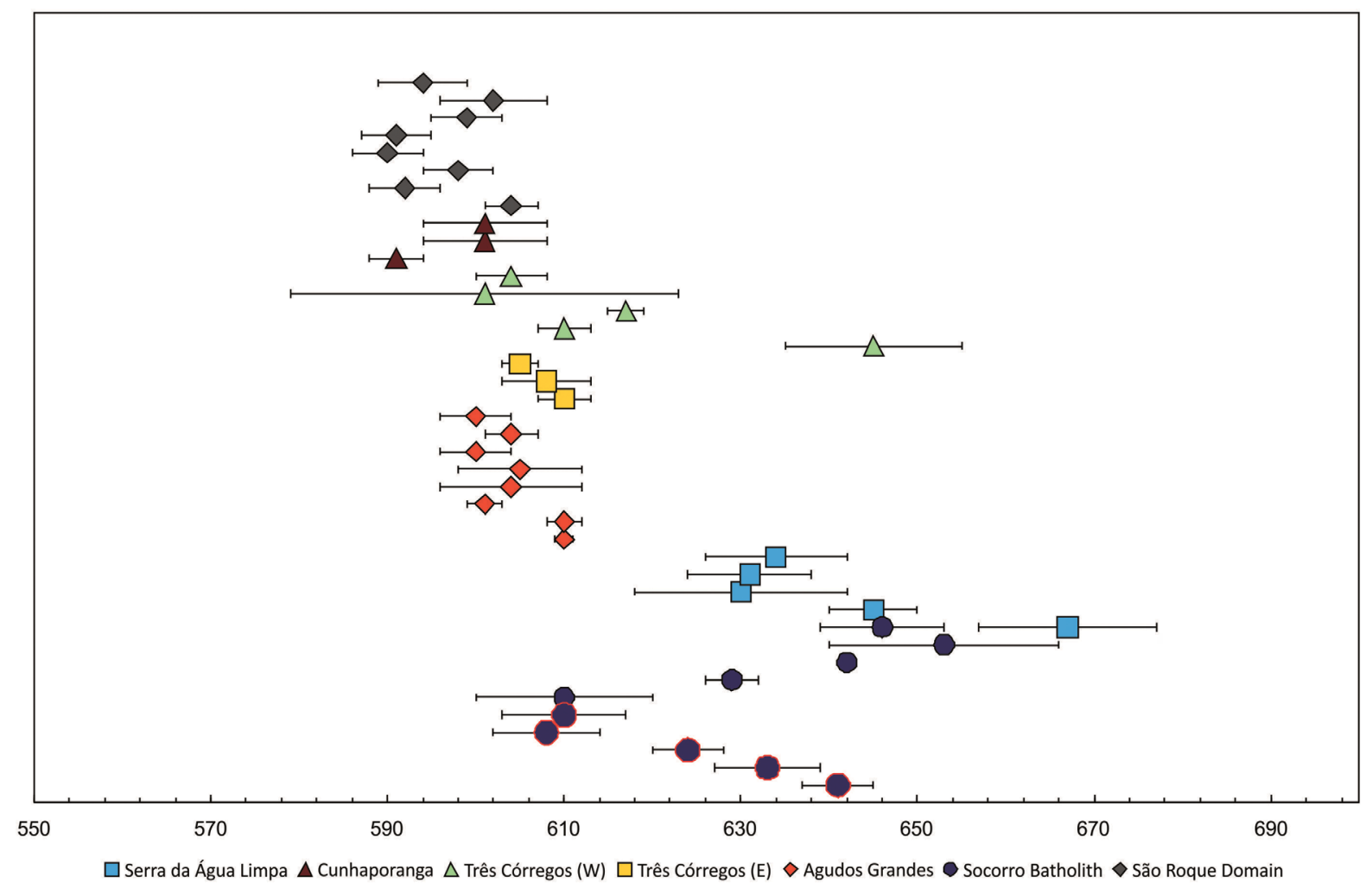

Figure 9. Timing of granite magmatism in Socorro Batholith compared with other major granite batholiths Três Córregos, Cunhaporanga, Serra da Água Limpa, Agudos Grandes and São Roque Domain. Data sources and names of the occurrences in Table 4. 
Table 4. Results of U-Pb isotope determinations of Três Córregos, Cunhaporanga, Serra da Água Limpa, Agudos Grandes and São Roque Domain.

\begin{tabular}{|c|c|c|c|c|c|c|c|}
\hline Sample & Unit & Rock type & Mineral & $\begin{array}{l}\text { Analytical } \\
\text { Method }\end{array}$ & Age & Error & Reference \\
\hline \multicolumn{8}{|l|}{ Socorro Batholith } \\
\hline 1. BRP - 12 & $\begin{array}{c}\text { Socorro } \\
\text { Charnockite }\end{array}$ & Charnockito & Zircon & SHRIMP & 641 & 4 & This study \\
\hline 2. ATB - 08 & $\begin{array}{c}\text { Atibaia } \\
\text { Charnockite }\end{array}$ & Charnockito & Zircon & SHRIMP & 633 & 6 & This study \\
\hline 3. BRP - 03 & Salmão & Bt syenogranite & Zircon & SHRIMP & 624 & 4 & This study \\
\hline 4. ATB - 13 & $\begin{array}{l}\text { Bragança } \\
\text { Paulista }\end{array}$ & Bt syenogranite & Zircon & SHRIMP & 608 & 6 & This study \\
\hline 5. $\mathrm{BRP}-08$ & $\begin{array}{l}\text { Bragança } \\
\text { Paulista }\end{array}$ & Hbl-bt granite & Zircon & SHRIMP & 610 & 7 & This study \\
\hline 6. H3 & $\begin{array}{l}\text { Bragança } \\
\text { Paulista }\end{array}$ & Granite & Zircon & TIMS & 610 & 10 & Ebert et al. 1996 \\
\hline 7. & $\begin{array}{l}\text { Bragança } \\
\text { Paulista }\end{array}$ & Granite & Zircon & TIMS & 629 & 3 & Topfner 1996 \\
\hline 8. H621 & Piracaia & $\begin{array}{c}\text { Milonitic } \\
\text { granitic gnaisse }\end{array}$ & Zircon & TIMS & 642 & 1 & $\begin{array}{l}\text { Hackspacher } \\
\text { et al. } 2003\end{array}$ \\
\hline 9. H705B & Piracaia & Orthogneiss & Zircon & TIMS & 653 & 13 & $\begin{array}{l}\text { Hackspacher } \\
\text { et al. } 2003\end{array}$ \\
\hline 10. H623A & $\begin{array}{l}\text { Paraisópolis } \\
\text { Complex }\end{array}$ & Granulite & Zircon & TIMS & 646 & 7 & $\begin{array}{l}\text { Hackspacher } \\
\text { et al. } 2003\end{array}$ \\
\hline \multicolumn{8}{|c|}{ Serra da Água Limpa } \\
\hline 10. RDTM 62 & Facies 3 & $\begin{array}{l}\text { Porphyritic } \\
\text { granite }\end{array}$ & Zircon & LA-ICPMS & 667 & 10 & Vinagre et al. 2014 \\
\hline 11. RDPA 44 (SALB) & Facies 3 & $\begin{array}{l}\text { Porphyritic } \\
\text { granite }\end{array}$ & Zircon & LA-ICPMS & 645 & 5 & Vinagre et al. 2014 \\
\hline 12. RDPA 46 (SALB) & Facies 3 & $\begin{array}{l}\text { Porphyritic } \\
\text { granite }\end{array}$ & Zircon & LA-ICPMS & 630 & 12 & Vinagre et al. 2014 \\
\hline 13. VAC 10 (SALB) & Facies 4 & $\begin{array}{l}\text { Porphyritic } \\
\text { granite }\end{array}$ & Zircon & LA-ICPMS & 631 & 7 & Vinagre et al. 2014 \\
\hline 14. RDIT 41 (SALB) & Facies 5 & Qtz-syenite & Zircon & LA-ICPMS & 634 & 8 & Vinagre et al. 2014 \\
\hline \multicolumn{8}{|l|}{ Agudos Grandes } \\
\hline 15. PD - $313 f$ & Turvo & $\begin{array}{c}\text { Ms-Bt } \\
\text { leucogranite }\end{array}$ & Monazite & TIMS & 610 & 1 & Janasi et al. 2001 \\
\hline 16. PD - 526 & Ibiúna & $\begin{array}{c}\mathrm{Hbl-Bt} \\
\text { monzogranite }\end{array}$ & Zircon & TIMS & 610 & 2 & Janasi et al. 2001 \\
\hline 17.PD - 462 & Piedade & Ms-Bt granite & Monazite & TIMS & 601 & 2 & Janasi et al. 2001 \\
\hline 18. PD - 474b & Piedade & Bt granite & Zircon & TIMS & 604 & 8 & Janasi et al. 2001 \\
\hline 19.PD - 420 & Piedade & Bt granite & Zircon & TIMS & 605 & 7 & Janasi et al. 2001 \\
\hline 20.PD - 498 & Roseira & Bt granite & Monazite & TIMS & 600 & 4 & Leite et al. 2007 \\
\hline 21.PD - 2266 & Serra dos Lopes & Bt granite & Monazite & TIMS & 604 & 3 & Leite et al. 2007 \\
\hline 22.PD - 2154 & Pilar do Sul & Ms-Bt granite & Monazite & TIMS & 600 & 4 & Leite et al. 2007 \\
\hline \multicolumn{8}{|l|}{ Três Córregos (E) } \\
\hline 23.E - 3 & Barra do Chapéu & Foliated granite & Zircon & TIMS & 610 & 3 & $\begin{array}{l}\text { Gimenez Filho } \\
\text { et al. } 2000\end{array}$ \\
\hline 24.MN - 15 & Barra do Chapéu & $\begin{array}{c}\text { Hbl-Bt } \\
\text { orthogneiss }\end{array}$ & Zircon & TIMS & 608 & 5 & $\begin{array}{l}\text { Gimenez Filho } \\
\text { et al. } 2000\end{array}$ \\
\hline 25.S - 1 & Saival & Hbl-Bt granite & Zircon & TIMS & 605 & 2 & $\begin{array}{l}\text { Gimenez Filho } \\
\text { et al. } 2000\end{array}$ \\
\hline
\end{tabular}


Bárbara Bueno Toledo et al.

Table 4. Continuation.

\begin{tabular}{|c|c|c|c|c|c|c|c|}
\hline Sample & Unit & Rock type & Mineral & $\begin{array}{l}\text { Analytical } \\
\text { Method }\end{array}$ & Age & Error & Reference \\
\hline \multicolumn{8}{|c|}{ Três Córregos (W) } \\
\hline 26. HP - 06 & Paina & Hbl-Bt tonalite & Zircon & SHRIMP & 645 & 10 & $\begin{array}{c}\text { Prazeres Filho } \\
2005\end{array}$ \\
\hline 27.HP - 07 & $\begin{array}{l}\text { Arrieiros - } \\
\text { Cerro Azul }\end{array}$ & $\begin{array}{c}\text { Hbl-Bt } \\
\text { granodiorite }\end{array}$ & Zircon & SHRIMP & 610 & 3 & $\begin{array}{c}\text { Prazeres Filho } \\
2005\end{array}$ \\
\hline 28.HP - 121 & $\begin{array}{l}\text { Arrieiros - } \\
\text { Cerro Azul }\end{array}$ & Granodiorite & Zircon & TIMS & 617 & 2 & $\begin{array}{l}\text { Prazeres Filho } \\
\text { et al. } 2003\end{array}$ \\
\hline 29.HP - 44 & São Sebastião & $\begin{array}{l}\text { Hbl-Bt qtz } \\
\text { monzonite }\end{array}$ & Zircon & SHRIMP & 601 & 22 & $\begin{array}{l}\text { Prazeres Filho } \\
2005\end{array}$ \\
\hline 30.HP - 44 & São Sebastião & $\begin{array}{l}\text { Hbl-Bt qtz } \\
\text { monzonite }\end{array}$ & Zircon & TIMS & 604 & 4 & $\begin{array}{l}\text { Prazeres Filho } \\
\text { et al. } 2003\end{array}$ \\
\hline \multicolumn{8}{|c|}{ Cunhaporanga } \\
\hline 31.HP - 03 & Ribeirão Butiá & Monzogranite & Zircon & TIMS & 591 & 3 & $\begin{array}{c}\text { Prazeres Filho } \\
2006\end{array}$ \\
\hline 32.HP - 437 & $\begin{array}{c}\text { Ribeirão } \\
\text { Butiá-Pitangui }\end{array}$ & $\begin{array}{c}\text { Qtz- } \\
\text { monzodiorite }\end{array}$ & Zircon & SHRIMP & 601 & 7 & $\begin{array}{c}\text { Prazeres Filho } \\
2006\end{array}$ \\
\hline 33.HP - 21 & Piraí do Sul & Monzogranite & Zircon & TIMS & 601 & 7 & $\begin{array}{l}\text { Prazeres Filho } \\
\text { et al. } 2003\end{array}$ \\
\hline \multicolumn{8}{|c|}{ São Roque Domain } \\
\hline 34. SR -07 & São Roque & Monzogranite & Zircon & SHRIMP & 604 & 3 & Janasi et al. 2016 \\
\hline 35.MD42B & Cantareira & Syenogranite & Zircon & SHRIMP & 592 & 4 & Janasi et al. 2016 \\
\hline 36.MD24 & Fazenda Itahyê & Monzogranite & Zircon & SHRIMP & 598 & 4 & Janasi et al. 2016 \\
\hline 37. JP-18 & $\begin{array}{l}\text { Vila dos } \\
\text { Remédios }\end{array}$ & Qtz-syenite & Zircon & SHRIMP & 590 & 4 & Janasi et al. 2016 \\
\hline 38. TICO-2 & Tico-Tico & Granite & Zircon & LA-ICPMS & 591 & 4 & Janasi et al. 2016 \\
\hline 39. CANT-6 & Cantareira & Granite & Zircon & LA-ICPMS & 599 & 4 & Janasi et al. 2016 \\
\hline 40.TAP-1 & Taipas & Granite & Zircon & LA-ICPMS & 602 & 6 & Janasi et al. 2016 \\
\hline 41.ITQ-2 & Itaqui & Granite & Zircon & LA-ICPMS & 594 & 5 & Janasi et al. 2016 \\
\hline
\end{tabular}

SHRIMP: Sensitive High Resolution Ion Microprobe ; TIMS: Thermal Ionization Mass Spectrometry ; LA-ICPMS: laser ablation inductively coupled plasma mass spectrometry.

water-fluxed regime (Martins 2006). It can therefore be speculated that the intrusion of large volumes of high-temperature, relatively primitive, HKCA granites may be in part responsible for the influx of heat and volatiles that facilitated widespread melting in the upper levels of the presently exposed crustal section of the SGN. The young ages of these Bragança Paulista-type granites seem difficult to reconcile with models that admit a pre-collisional setting for the HKCA magmatism, and indeed high-pressure metamorphism diagnostic of collisional setting is, at least in part, older (Reno et al. 2009). The evolved isotope signature and enriched geochemical signature of these HKCA granites is indicative that they are, at least in part, the products of crustal recycling. The few inherited zircon cores identified in this work show $-1.4-1.8 \mathrm{Ga}$ ages that overlap the $\mathrm{Sm}-\mathrm{Nd}$ model ages reported for the SGN (e.g., Janasi 1999), which is consistent with the idea that the reworked crust is distinct, and younger, than that forming the nearby Apiaí and São Roque Domains in the Ribeira Belt.

\section{CONCLUSIONS}

Our U-Pb zircon SHRIMP dating program determined the ages of the main components of the Socorro Batholith, a major component of the allochtonous Socorro-Guaxupé Nappe. Two samples of HKCA porphyritic biotite-hornblende granites of relatively primitive $\left(60-67 \mathrm{wt} \% \mathrm{SiO}_{2}\right)$ that are the most voluminous component of the batholith were dated, and yielded similar results (Pedra Bela sample BRP-08 in the northern portion of the batholith, $610.1 \pm 7.0 \mathrm{Ma}$ and Bragança Paulista sample ATB-13 in the southern portion, $608.3 \pm$ 6.6 Ma). These ages are similar to U-Pb zircon ages recently obtained by in situ methods in similar rocks (HKCA granites) in other large batholiths in neighboring domains (Apiaí 
and São Roque) from the Ribeira Belt, but younger than the $\mathrm{U}-\mathrm{Pb}$ zircon LA-ICPMS ages reported for the HKCA granites of the Serra da Água Limpa Batholith, located immediately east of the Socorro batholith (645-630 Ma).

A more fractioned ( $\left.>72 \mathrm{wt} \% \mathrm{SiO}_{2}\right)$ "Salmão-type" granite reported in the literature as related to a younger event ("Socorro II magmatism") yielded a precise age that is clearly older $(624.4 \pm 3.6 \mathrm{Ma})$ than the two HKCA samples. This age is similar to that of the oldest anatectic granites and migmatites that were produced during a prolonged period of high-grade metamorphism (635-605 Ma) that affected the SGN. Our data thus indicates that at least part of the HKCA magmatism of the Socorro batholith post-dates the high-P metamorphism associated to continental collision, and may have been a source of heat and volatiles to the high-T metamorphism responsible for partial melting of the upper portions of the crustal section represented by the SGN.

Two charnockitic rocks that show transitional contacts with granites of the Socorro batholith were also dated. The Socorro Charnockite has an age of $641.6 \pm 4.1 \mathrm{Ma}$, which overlaps those of regional orthogneisses (in part also of charnockitic character) considered as associated with pre-collisional tectonics (subduction-related?). However, it is reported to transition to granites that are very similar to the HKCA granites of the Socorro Batholith, which are yet undated. The Atibaia Charnockite is younger $(633.3 \pm 6.2 \mathrm{Ma})$ and has distinct geochemical affinity (lower mg\# and $\mathrm{Sr}$ content; higher $\mathrm{Zr}$ ), which may signal a different tectonic setting at the end of the period of plate consumption as yet poorly characterized.

\section{ACKNOWLEDGEMENTS}

Financial support for this work was provided by Fapesp through Grant 2015/01817-6 (to VAJ). LGRS acknowledges a Scientific Initiation scholarship by CNPq. VAJ is a CNPq Productivity Researcher (Grant 305661/2014-0). Kei Sato at CPGeo is acknowledged for qualified support in obtaining and processing geochronological data. A careful review by Claudio Valeriano, Stefano Zincone, Rodrigo Vinagre and the associated editor Umberto Cordani were very helpful to improve the manuscript.

\section{SUPPLEMENTARY DATA}

Supplementary data associated with this article can be found in the online version: Supplementary data A1-A5.

\section{REFERENCES}

Almeida V.V., Janasi V.A., Heaman L.M., Shaulis B.J., Hollanda M.H.B.M., Renne P.R. 2018. Contemporaneous alkaline and tholeiitic magmatism in the Ponta Grossa Arch, Paraná-Etendeka Magmatic Province: Constraints from U-Pb zircon/baddeleyite and $40 \mathrm{Ar} / 39$ Ar phlogopite dating of the José Fernandes Gabbro and mafic dykes. Journal of Volcanology and Geothermal Research, 355:55-65. http:// dx.doi.org/10.1016/j.jvolgeores.2017.01.018

Artur A.C. 2003. Complexo Granitóide Plurisserial Socorro: Geologia, petrologia e recursos minerais. Livre-Docência Thesis, UNESP, Rio Claro, SP, 139 pp.

Artur A.C., Wernick E., Galembeck T.M.B. 1996. O Complexo Plurisserial Socorro (SP/MG): Um Indicador Geodinâmico Brasiliano. In: $39^{\circ}$ Congresso Brasileiro de Geologia, Salvador. Resumo Expandido, 6:14-16.

Artur A.C., Wernick E., Hôrmann P.K., Weber-Diefenbach K. 1993. Associações plutônicas do Complexo Granitóide Socorro (Estados de São Paulo e Minas Gerais, SE Brasil). Revista Brasileira de Geociências, 23(3):265-273.

Artur A.C., Wernick E., Souza S.C.A. de, Weber-Diefenbach K. 1994. Tipologia de zircão e quimismo do complexo monzoníticomonzodiorítico Piracaia, SP. Geociências, 13(2):167-173.

Barbarin B. 1999. A review of the relationships between granitoid types, their origins and their geodynamic environments. Lithos, 46(3):605-626. https://doi.org/10.1016/S0024-4937(98)00085-1

Black L.P., Kamo S.L., Allen C.M., Davis D.W., Aleinikoff J.N., Valley J.W., Mundil R., Campbell I.H., Korsch R.J., Williams I.S., Foulis C.
2004. Improved $206 \mathrm{~Pb} / 238 \mathrm{U}$ microprobe geochronology by the monitoring of a trace-element related matrix effect; SHRIMP, IDTIMS, ELA-ICP-MS and oxygen isotope documentation for a series of zircon standards. Chemical Geology, 205(1-2):115-140.

Boynton W.V. 1984. Cosmochemistry of the rare earth elements: meteorite studies. In: Henderson P. (Ed.), Rare Earth Elemen Geochemistry, pp.63e114.

Campos Neto M.C., Basei M.A.S., Alves F.R., Figueiredo M.C.H. 1984a. Geologia da Folha Bragança Paulista - 1:50.000. Relatório Final. São Paulo, Convênio SICCT/PRÓMINÉRIO-IG/USP. 162 p.

Campos Neto M.C., Basei M.A.S., Alves F.R., Vasconcelos A.C.B. 1984 b. A Nappe de cavalgamento Socorro (SP-MG). In: $33^{\circ}$ Congresso Brasileiro de Geologia, Rio de Janeiro. Anais, 4:1809-1822.

Campos Neto M.C., Caby R. 2000. Terrane accretion and upward extrusion of high-pressure granulites in the Neoproterozoic nappes of southeast Brazil: Petrologic and structural constraints. Tectonics, 19(4):669-687. https://doi.org/10.1029/1999TC900065

Ebert H.D., Chemale F., Babinski M., Artur A.C., Van Schmus W.R. 1996. Tectonic setting and U/Pb zircon dating of the plutonic Socorro Complex in the Transpressive Rio Paraíba do Sul Shear Belt, SE Brazil. Tectonics, 15(3):688-699. http://dx.doi.org/10.1029/95TC03247

Gimenez Filho A., Janasi V.A., Campanha G.A.C., Teixeira W., Trevizoli Júnior L.E. 2000. U-Pb dating and Rb-Sr isotope geochemistry of the eastern portion of the Três Córregos Batholilth, Ribeira Fold Belt, São Paulo. Revista Brasileira de Geociências, 30(1):45-50. 
Hackspacher P.C., Fetter A.H., Ebert H.D., Janasi V.D.A., Dantas E.L., de Oliveira M.A.F., Braga I.F., Negri F.D.A. 2003. Magmatismo há 660-640 Ma no Domínio Socorro: registros de convergência pré-colisional na aglutinação do Gondwana Ocidental. Geologia USP- Série Científica, 3(1):85-96. https://doi.org/10.5327/ S1519-874X2003000100007

Haddad R.C. 1995. O batólito granitóide Pinhal-Ipuiúna (SP-MG): um exemplo do magmatismo cálcio-alcalino potássico neoproterozóico no sudeste brasileiro. PhD Thesis, Universidade de São Paulo, São Paulo, $261 \mathrm{p}$.

Heilbron M., Pedrosa-Soares A.C., Campos Neto M.C., da Silva L.C., Trouw R.A.J., Janasi V.A. 2004. A Província Mantiqueira. In: MantessoNeto V., Bartorelli A., Brito Neves B.B. (Eds.). Geologia do Continente Sul-americano: Evolução da Obra de Fernando Flávio Marques de Almeida. São Paulo, Beca, p. 203-234.

Heilbron M., Ribeiro A., Valeriano C.M., Paciullo F.V., Almeida J.C.H., Trow R.J.A., Tupinambá M., Eirado L.G. 2017. Ribeira Belt. In: Heilbron M., Cordani U.G., Alkmim F.F. (Eds.). San Francisco Craton, Eastern Brazil. London, Springer, p. 277-302, 331 p.

Heilbron M., Tupinambá M., Valeriano C.M., Armstrong R., do Eirado Siva L.G., Melo R.S., Simonetti A., Pedrosa-Soares A.C., Machado N. 2013. The Serra da Bolívia complex: The record of a new Neoproterozoic arc-related unit at Ribeira belt. Precambrian Research, 238:158-175. http://dx.doi.org/10.1016/j. precamres.2013.09.014

Janasi V.A. 1999. Petrogênese de granitos crustais na Nappe de Empurrão Socorro-Guaxupé (SP-MG): uma contribuição da geoquímica elemental e isotópica. Post-Doctoral Thesis, Universidade de São Paulo, São Paulo, 304 p.

Janasi V.A. 2002. Elemental and Sr-Nd isotope geochemistry of two Neoproterozoic mangerite suites in SE Brazil: implications for the origin of the mangerite-charnockite-granite series. Precambrian Research, 119(1):301-327. http://dx.doi.org/10.1016/ S0301-9268(02)00127-4

Janasi V.A., Andrade S., Vasconcellos A.C.B.C., Henrique-Pinto R., Ulbrich H.H.G.J. 2016. Timing and sources of granite magmatism in the Ribeira Belt, SE Brazil: Insights from zircon in situ U-Pb dating and Hf isotope geochemistry in granites from the São Roque Domain. Journal of South American Earth Sciences, 68:224-247. http://dx.doi.org/10.1016/j.jsames.2015.11.009

Janasi V.A., Leite R.J., van Schmus W.R. 2001. U-Pb chronostratigraphy of the granitic magmatism in the Agudos Grandes Batholith (west of Sao Paulo, Brazil) -- implications for the evolution of the Ribeira Belt. Journal of South American Earth Sciences, 14(4):363-376. http://dx.doi.org/10.1016/S0895-9811(01)00034-7

Janasi V.A., Ulbrich H.H.G.J. 1991. Late Proterozoic granitoid magmatism in the State of SÃ£o Paulo, southeastern Brazil. Precambrian Research, 51(1-4):351-374. https://doi. org/10.1016/0301-9268(91)90108-M

Janasi V.A., Vlach S.R.F., Campos Neto M.C., Ulbrich H.H.G.J. 2009. Associated A-type subalkaline and high-K calc-alkaline granites in the Itu Granite Province, Southeastern Brazil: Petrological and tectonic significance. Canadian Mineralogist, 47(6):1505-1526. http://dx.doi.org/10.3749/canmin.47.6.1505

Leite R.J., Heaman L.M., Janasi V.A., Martins L., Creaser R.A. 2007. The late- to postorogenic transition in the Neoproterozoic Agudos Grandes Granite Batholith (Apiai Domain, SE Brazil): Constraints from geology, mineralogy, and U-Pb geochronology. Journal of South American Earth Sciences, 23(2-3):193-212. http://dx.doi. org/10.1016/j.jsames.2006.09.022

Ludwig K. 2003. User's manual for isoplot/Ex version 3.00, a geochronological toolkit for Microsoft Excel. Berkley Geochronology Center Special Publications, 4, 72pp.
Ludwig K. 2009. SQUID 2; A User's Manual. Berkely Geochronology Center Special Publications, 5, 110p

Mantovani M.S.M., Brito Neves B.B. 2005. The Paranapanema Lithospheric Block: Its Importance for Proterozoic (Rodinia, Gondwana) Supercontinent Theories. Gondwana Research, 8(3):303315. https://doi.org/10.1016/S1342-937X(05)71137-0

Martins L. 2006. Geração e migração de magmas graníticos na crosta continental: estudos de detalhe em granitos e migmatitos da região de Nazaré Paulista (SP). PhD Thesis, Universidade de São Paulo, São Paulo, 159 p.

Martins L., Vlach S.R.F., Janasi V.A. 2009. Reaction microtextures of monazite: Correlation between chemical and age domains in the Nazaré Paulista migmatite, SE Brazil. Chemical Geology, 261(34):271-285. http://dx.doi.org/10.1016/..chemgeo.2008.09.020

Meira V.T., García-Casco A., Juliani C., Almeida R.P., Schorscher J.H.D. 2015. The role of intracontinental deformation in supercontinent assembly: insights from the Ribeira Belt, Southeastern Brazil (Neoproterozoic West Gondwana). Terra Nova, 27(3):206-217. https://doi.org/10.1111/ter.12149

Mori P.E., Reeves S., Correia C.T., Haukka M. 1999. Development of a fused glass disc XRF facility and comparison with the pressed powder pellet technique at Instituto de Geociências, Universidade de São Paulo. Revista Brasileira de Geociências, 29(3):441-446. http://doi.org/10.5327/rbg.v29i3.715

Navarro M.S., Andrade S., Ulbrich H., Gomes C.B., Girardi V.A.V. 2008. The Direct Determination of Rare Earth Elements in Basaltic and Related Rocks using ICP-MS: Testing the Efficiency of Microwave Oven Sample Decomposition Procedures. Geostandards and Geoanalytical Research, 32(2):167-180. https://doi. org/10.1111/j.1751-908X.2008.00840.x

Oliveira M.A.F., Morales N., Fúlfaro V.J., Souza Filho E.E., Campos E.G., Godoy A.M., Simões L.S.A., Artur A.C., Rueda J.R.J., Batista J.J., Angeli N. 1994. Geologia da Quadrícula de Atibaia, IGCE/UNESP.

Prazeres Filho H.J. 2006. Caracterização geológica e petrogenética do batólito granítico Três Córregos (PR-SP): geoquímica isotópica (Nd$\mathrm{Sr}-\mathrm{Pb}$ ), idades (ID-TIMS/SHRIMP) e 'delta18'O em zircão. PhD Thesis, Universidade de São Paulo, São Paulo, 207pp.

Prazeres Filho H.J., Harara O.M., Basei M.A.S., Passarelli C.R., Siga Jr. O. 2003. Litoquímica, geocronologia U-Pb e geologia isotópica (Sr-Nd-Pb) das rochas graníticas dos batólitos Cunhaporanga e Três Córregos na porção sul do Cinturão Ribeira, Estado do Paraná Geologia USP- Série Científica, 3:51-70. https://doi.org/10.5327/ S1519-874X2003000100005

Reno B.L., Brown M., Kobayashi K., Nakamura E., Piccoli P.M., Trouw R.A.J. 2009. Eclogite-high-pressure granulite metamorphism records early collision in West Gondwana: new data from the Southern Brasília Belt, Brazil. Journal of the Geological Society, 166(6):10131032. https://doi.org/10.1144/0016-76492008-140

Rocha B.C., Moraes R., Möller A., Cioff C.R. 2018. Magmatic inheritance vs. UHT metamorphism: Zircon petrochronology of granulites and petrogenesis of charnockitic leucosomes of the Socorro-Guaxupé nappe, SE Brazil. Lithos, 314-315:16-39. http:// dx.doi.org/10.1016/j.lithos.2018.05.014

Rocha B.C., Moraes R., Möller A., Cioff C.R., Jercinovic M.J. 2017 Timing of anatexis and melt crystallization in the Socorro-Guaxupé Nappe, SE Brazil: Insights from trace element composition of zircon, monazite and garnet coupled to UPb geochronology. Lithos, 277:337-355. http://dx.doi.org/10.1016/j.lithos.2016.05.020

Sato K., Tassinari C.C.G., Basei M.A.S., Siga Jr. O., Onoe A.T., Souza M.D. 2014. Sensitive High Resolution Ion Microprobe (SHRIMP IIe/MC) of the Institute of Geosciences of the University of São Paulo, Brazil: analytical method and first results. Geologia USP, Série Científica, 14(3):3-18. 
Stacey J.S., Kramer J.D. 1975. Approximation of terrestrial lead isotope evolution by a two-stage model. Earth and Planetary Science Letters, 26:207-221.

Tassinari C. 1988. As idades das rochas e dos eventos metamórficos da porção sudeste do Estado de São Paulo e sua evolução crustal. PhD Thesis, Universidade de São Paulo, São Paulo, 236 p.

Topfner C. 1996. Brasiliano-granitoide in den Bundesstaaten São Paulo und Minas Gerais, Brasilieneine Vergleichende studie. Münchner, Münchner Geologische Hefte, v.17.

Trouw R.A.J., Peternel R., Ribeiro A., Heilbron M., Vinagre R., Teixeira P.D., Trouw C.C., Fontainha M., Kussama H.H. 2013. A new interpretation for the interference zone between the Southern Brasília Belt and the Central Ribeira Belt, SE Brazil. Journal of South American Earth Sciences, 48:43-57. http://dx.doi.org/10.1016/j.jsames.2013.07.012

Valeriano C.M., Mendes J.C., Tupinambá M., Bongiolo E., Heilbron M., Junho M.C.B. 2016. Cambro-Ordovician post-collisional granites of the Ribeira belt, SE-Brazil: A case of terminal magmatism of a hot orogen. Journal of South American Earth Sciences, 68:269-281. https://doi.org/10.1016/j.jsames.2015.12.014
Vinagre R., Trouw R.A.J., Mendes J.C., Teixeira P.D., Peternel R., Matos G. 2014. New evidence of a magmatic arc in the southern Brasília Belt, Brazil: The Serra da Água Limpa batholith (Socorro-Guaxupé Nappe). Journal of South American Earth Sciences, 54:120-139. http://dx.doi.org/10.1016/j.jsames.2014.05.002

Wernick E., Artur A.C., Hörmann P.K., Weber-Diefenbach K., Fahl F.C. 1997. O magmatismo Piracaia, SP (SE Brasil): aspectos composicionais e evolutivos. Revista Brasileira de Geociências, 27(1):53-66.

Wernick E., Didier J., Artur A.C., Hörmann P.K. 1984a. Caracterização da zona marginal charnockítica do Complexo Socorro nos arredores da cidade homônima, SP/MG. 33 Congresso Brasileiro de Geologia, 6:2919-2934.

Wernick E., Hörmann P., Artur A.C., Eulert H. 1984b. Aspectos petrológicos do Complexo Granítico Socorro (SP/MG): dados analíticos e discussão preliminar. Revista Brasileira de Geociências, 14(1):23-29.

Wernick E., Menezes A.C. 2001. The Late Precambrian K-alkaline magmatism in the Ribeira Fold Belt: a case study of the Piracaia pluton, State of São Paulo, SE Brasil, and its potential mineralization (Cu, Zn, Gd). Journal of Asian Earth Sciences, 19(3):347-373. http:// dx.doi.org/10.1016/S1367-9120(00)00027-4 